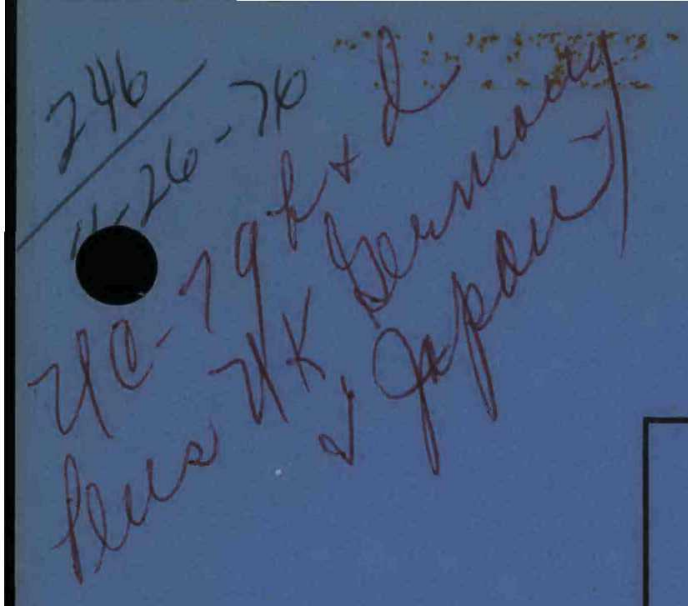
STATUS OF GAMMA-RAY HEATING CHARACTERIZATION

IN LMFBR

$$
\begin{aligned}
& \text { HEDL-TME 75-126 } \\
& \text { UC-79b, d }
\end{aligned}
$$

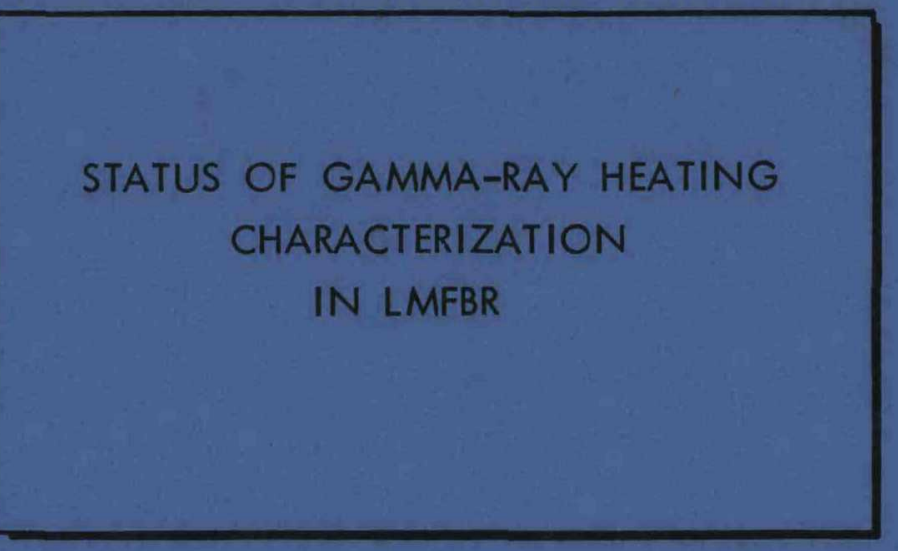

i)

、

HANFORD ENGINEERING DEVELOPMENT LABORATORY

P.O. Box 1970 Richland, WA 99352

Operated by Westinghouse Hanford Company

A Subsidiary of Westinghouse Electric Corporation

Prepared for the U.S. Energy Research and Development Administration under Contract No. E(45-1)-2170 


\section{DISCLAIMER}

This report was prepared as an account of work sponsored by an agency of the United States Government. Neither the United States Government nor any agency Thereof, nor any of their employees, makes any warranty, express or implied, or assumes any legal liability or responsibility for the accuracy, completeness, or usefulness of any information, apparatus, product, or process disclosed, or represents that its use would not infringe privately owned rights. Reference herein to any specific commercial product, process, or service by trade name, trademark, manufacturer, or otherwise does not necessarily constitute or imply its endorsement, recommendation, or favoring by the United States Government or any agency thereof. The views and opinions of authors expressed herein do not necessarily state or reflect those of the United States Government or any agency thereof. 


\section{DISCLAIMER}

Portions of this document may be illegible in electronic image products. Images are produced from the best available original document. 


\section{NOTICE}

This report was prepared as an account of work sponsored by the United States Government. Neither the United States nor the U.S. ERDA, nor any of their employees, nor any of their contractors, subcontroctors, or their employees, makes any warranty, express or implied, or assumes any legal liability or responsibility for the accuracy, completeness or usefulness of any information, apparatus, product or process disclosed, or represents that its use would not infringe privately owned rights.

Printed in the United States of America

$$
\text { Available from }
$$

National Technical Information Service

U.S. Department of Commerce 5285 Port Royal Road

Springfield, Virginia 22161

Price: Printed Copy $\$ 5.50$; Microfiche $\$ 2.25$ 
HEDL-TME 75-126

UC-7\% , d

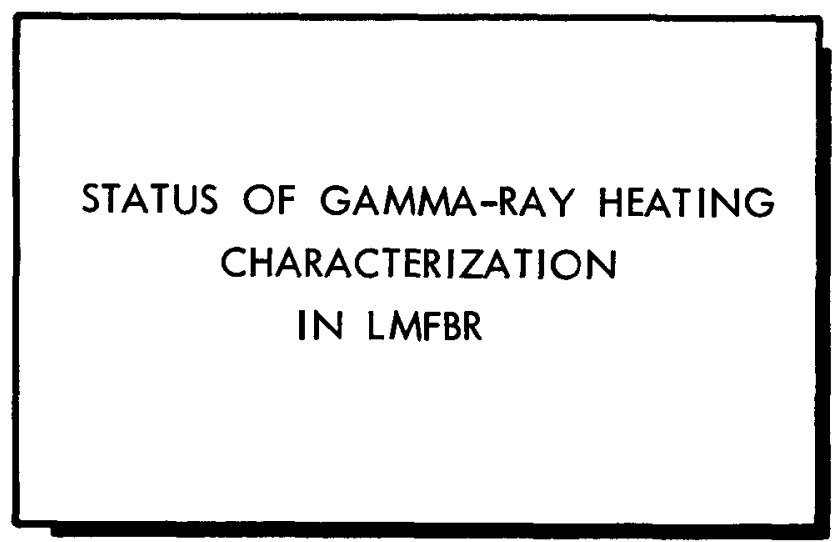

Raymond Gold

November, 1975

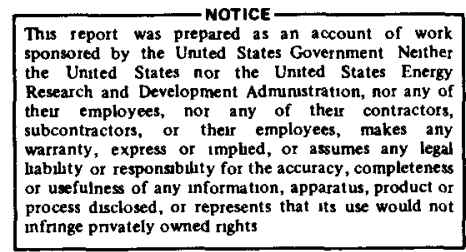

\section{Hanford Engineering Development Laboratory}

Operated by the

Westinghouse

Hanford Company
A Subsidiary of Westinghouse Electric Corporation for the United Stotes

Energy Resealreh and

Development Administration

Contract No. E(45-1)2170 
○

.

- 


\title{
STATUS OF GAMMA-RAY HEATING CHARACTERIZATION IN LMFBR
}

\author{
Raymond Gold*
}

\begin{abstract}
$\underline{\text { ABSTRACT }}$
Efforts to define gama-ray heating in Liquid Metal Fast Breeder Reactor (LMFBR) environments have been surveyed. Emphasis is placed on both current practice for the Experimental Breeder Reactor-II (EBR-II) and future needs of the Fast Flux Test Facility (FFTF). Experimental and theoretical work are included in this preliminary survey for both high and low power environments. Current "stateof-the-art" accuracies and limitations are assessed. on this basis, it is concluded that a broad and sustained effort be initiated to meet requested FFTF goal accuracies. To this end, recommendations are advanced for improving the current status of gamma heating characterization and temperature measurements in LMFBR.
\end{abstract}

*Radiation and Dosimetry Services, Inc., Richland, WA 99352. 
"without proper experiments I conclude nothing"

Johann Kepler (1571-1630) 


\section{CONTENTS}

\section{Page}

I. INTRODUCTION

II. EXPERIMENTAL METHODS 8

A. High Power Environments 8

1. Active Measurements 8

2. Passive Measurements 12

B. Low Power Environments 20

1. Gamma-Ray Dosimetry 21

2. Gamma-Ray Spectrometry 25

III. CALCULATIONS 29

IV. RECOMMENDATIONS 40

A. Development of Experimental Techniques 41

1. Calorimetry 41

2. Ionization Chambers 42

3. TED Monitors 42

4. VAP Monitors 43

5. TLD Dosimeters 43

6. Compton Recoil Gamma-Ray Spectroscopy 43

B. Development of Calculational Methods 44

1. Basic Nuclear Data 44

2. Analytical Techniques 45

C. Specific Experiments 46

1. In-Core Gamma-Ray Spectrometry 46

2. Gamma-Ray Production Measurements 47

V. SUMMARY 49

VI. REFERENCES 51 
1. Calorimeter in Current Use at EBR-II for Active Measurement of Gamma (and Neutron) Heating

2. Design of Passive Vapor Pressure (VAP) Monitor for In-Core Temperature Measurements

3. Passive Thermal Expansion Difference (TED) Monitor in Current Use at EBR-II

4. Passive Gamma Expansion Difference (GEDM) Monitor Under Test at EBR-II

5. The Gamma-Ray Energy Distribution at the Core Center of a Low Power Fast Critical Facility (ZPR-6, Assembly 6) Obtained by Compton Recoil Gamma-Ray Spectroscopy

6. Generalized Block Diagram of Gamma Calculations

\section{TABLES}

Table

Page

I. Neutron and Gamma Uncertainties in LMFBR 3

II. Priority Matrix for Materials Irradiation Tests 5

III. Gamma Heating Classification for Materials Irradiation 6

IV. Qualitative Comparison of Passive Temperature Monitors 18

V. Gamma-Ray Computer Codes 32

VI. Accuracy of Selected LMFBR Gamma Heating Activities 50 


\section{STATUS OF GAMMA-RAY HEATING CHARACTERIZATION \\ IN LMFBR}

\section{INTRODUCTION}

Many extensive programmatic efforts have been supported over the years in order to define the equilibrium neutron radiation component which exists in nuclear (fission) reactors. As a result of these efforts, a sound bas is exists for predicting the in-core neutron spectrum in a wide variety of reactor environments. (1) To attain this capability, dedicated long-range programs have been required covering diverse theoretical and experimental activities. Measurements and calculations, at both high and low power, have been conducted in many specialized facilities. In-core neutron dosimetry has been developed for high power reactor environments ${ }^{(2)}$ and applicability for Liquid Metal Fast Breeder Reactors (LMFBR) has been demonstrated by analysis of experiments carried out in EBR-II. (3) In spite of this progress, disagreements between calculation and experiment still arise and the present "state-of-the-art" accuracy for neutron flux and fluence has been assessed at approximately $5-10 \%(10)$, * with a requested future goal of $1-3 \%$. ${ }^{(4)}$ The difficulties encountered in attempts to provide absolute measurements at the $1-3 \%$ level have, however, been recognized and as a consequence more realistic goals of $2-5 \%$ have been projected for the immediate future.

In contrast with neutron dosimetry, US and European efforts to define the in-core gamma-ray component have been sporadic at best over the last two decades or more and have found marginal if any support. As a result, a serious deficiency has unfortunately been produced in overall nuclear reactor knowledge, since the radiation field found in reactors is comprised of two rather than just a single component, namely gamma-rays as well as neutrons. The interdependence of these two (neutral) particle components of the in-core reactor radiation field has already been touched upon ${ }^{(5)}$ and it is expected that future work will provide a more quantitative understanding of this fundamental relationship.

\footnotetext{
*Relative errors quoted throughout this survey refer to the lo level.
} 
Perhaps the earliest work in defining in-core gamma-ray heating and temperature distributions was expended for the Materials Test Reactor (MTR), in the early 1950's. (6-9) Here, accurate temperature profiles were desired for in-core thermal neutron materials irradiation experiments. While these efforts did provide a good starting point for gamma heat calorimetry, very limited effort was devoted to other experimental approaches and essentially no theoretical support was provided. After a lapse of many years, interest in gamma heating was rekindled in the early 1970's when EBR-II affected the transition from a demonstration LMFBR to an irradiation facility for high fluence test experiments. Just as for MTR, the motivation for EBR-II gamma heating characterization stems from the need to define the environment of materials tests more precisely. However, little programmatic support has been formalized for this specific task, with the possible exception of recent work by the EBR-II project. In spite of this severe handicap, considerable advances have been made by the EBR-II project in both calculational methods and experimental techniques. It is, therefore, entirely reasonable to assume that present efforts at EBR-II are a realistic description of the current "state-of-the-art" in reactor gamma-heating and/or temperature measurements as well as calculations. In this context, Table I contrasts the current accuracies obtainable for both neutron and gamma-ray components in EBR-II and the forecasted requirements for FFTF. (4) The difference in the "state-of-the-art" between neutron and gamma-ray core characterization is not fully reflected in Table I. While 10\% has been cited for the current gamma flux and gamma heat uncertainties in EBR-II, it can be argued that this assessment is over-optimistic. The difference in status of the gamma and neutron components can be more generally understood by comparing the overall effort required to attain requested FFTF goal accuracies from current (EBR-II) levels, since the latter uncertainties reflect the historical interest and support and underlying neutron dosimetry over the years as opposed to the extremely limited emphas is with regard to the gamma-ray component. Consequently, the broad foundation which already exists for the neutron component provides a legitimate basis for the projection of a $1-3 \%$ future uncertainty requirement for FFTF; whereas the very 
TABLE I

NEUTRON AND GAMMA UNCERTAINTIES IN LMFBR
$\begin{array}{cc}\text { Current Status } & \text { Future Requirements } \\ (\text { EBR-II) } & \text { (FFTF) }\end{array}$

(\%)

(\%)

Current Goals Requested Goals

Neutron Flux

Neutron Fluence

Gamma Flux

Gamma Heat
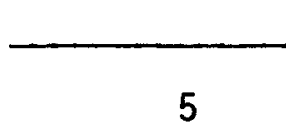

$5-10$

10

10
2-5

2-5

2-5

2-5
1-3

1-3

$<3$

$<3$

lack of a comparable basis for the gamma-ray component implies that the more modest goal of $<3 \%$ uncertainty is warranted under the presumption that considerably greater effort need necessarily be expended.

Optimum design of LMFBR is complex and dependent upon many factors, only one of which is gamma-heating. Adequate cooling for in-core structural components must be provided since these components are subjected to temperature gradients induced by gamma heating, which could otherwise produce differences in swelling and creep leading to bowing of subassembly (S/A) tubes and ducts. LMFBR design must therefore consider the need for core restraint mechanisms to prevent the occurrence of any such structural deformations. As a corollary, it is evident that gamma heating plays a major role in the design of LMFBR control and safety rods. In fact, recent concern with gamma heating in some LMFBR control rod concepts has supplied some impetus toward developing calculational models for the prediction of gamma heating $(10)$ and comparison of these predictions with low power experiments. (11) Further work should permit more quantitative evaluation of control schemes for advanced LMFBR designs. The gamma component of the in-core radiation field also plays a fundamental role in shielding design for LMFBR as well as cooling design for aftershutdown decay heat. Both of these safety related considerations have nontrivial impact on the overall cost of LMFBR. To this end, calculations and (low power) experiments have been conducted in both of these areas (12) which parallel efforts expended in LMFBR control rod development. 
As opposed to these general motivations for gamma heating characterization, there exist specific needs of workers using high power radiation test facilities which are mandatory for the proper interpretation of experiments. These needs are emphasized throughout this survey. From the viewpoint of these experimenters, the temperature $T$ of the given radiation damage experiment is a crucial variable. Many such experiments cannot be precisely analyzed or quantified without an accurate knowledge of the temperature history of the irradiation. In this context, it is the temperature induced by the in-core radiation field rather than the radiation field itself, which is of interest. Ideally, most workers desire the time-dependent temperature history, $T(t)$, over the complete course of the in-core exposure. Many workers would like $T(t)$ to be known to within $\pm 5^{\circ} \mathrm{F}$ over the range $600^{\circ} \mathrm{F} \delta \mathrm{T} \delta 1400^{\circ} \mathrm{F}$; moreover many imply that violation of the condition $T(t)=$ constant could compromise their experiments.

Although $T(t)$ is desired, the prediction of $T(t)$ at a given in-core location is based upon the solution of two complex (coupled) problems. One must first calculate the gamma heat (and in certain cases non-negligible neutron heating should also be included) and then use these results as the heat generation source in thermal-hydraulics calculations to predict temperature. Consequentiy, since $T$ is of paramount interest to the materials experimenter, gamma heating is indispensible in all design stages of the experiment. This survey tacitly assumes that more traditional thermalhydraulics calculations are on safer ground and therefore concentrates on the gamma heating aspects of these coupled problems. This assumption does not imply that review of thermal-hydraulics is unwarranted, but rather that it lies outside the scope of the present survey.

Materials irradiation tests can be classified according to the type of material under study and the specific observable being monitored. Consider the matrix of Table II as an illustrative means of categorizing these experiments. Associated with each possible test in the matrix of Table II is a level of priority which should be based upon the overall importance of the given matrix element to the optimum design (including economics, doubling time, safety, reliability, etc.) of LMFBR. (In Table II, undefined priority 
levels shown are for illustrative purposes only and in no way reflect actual decisions on priority levels.)

TABLE II

PRIORITY MATRIX FOR MATERIALS IRRADIATION TESTS

\begin{tabular}{|c|c|c|c|}
\hline & Fuels & Absorbers & $\begin{array}{l}\text { Structural } \\
\text { Materials }\end{array}$ \\
\hline Tensile Strength & 2 & 3 & 1 \\
\hline Irradiation Swelling & 2 & 2 & 1 \\
\hline Irradiation Creep & 1 & 2 & 2 \\
\hline Fracture Toughness & 3 & 4 & 2 \\
\hline Modulus of Rupture & 2 & 2 & 1 \\
\hline Residual Ductility & 2 & 3 & 2 \\
\hline
\end{tabular}

In this manner, a hierarchy can be established within the totality of all relevant LMFBR materials tests, which can be used in turn in allocating FFTF time and space to given experiments. Experiments of lower priority would generally be conducted with passive monitors, whereas high priority experiments would call for active monitors in instrumented S/A or closed loops (with heating and temperature control). These cannot be hard and fast operating rules, however, as other important criteria exist which are more intimately related to the given materials test, such as specimen size and geometry, sensitivity to temperature, total fluence required, etc. Consequently, the need for gamma heating information can be based upon the priority level (Table II) combined with certain characteristic requirements which are inherently demanded by the very nature of the specific experiment. Based on these considerations, Table III presents a recommended classification describing various degrees of need for gamma heating information in materials irradiations.

Certain high priority experiments may require little or negligible environmental perturbation for long irradiation periods. To satisfy such tests, it has been suggested that consideration be given to establishing a 
TABLE III

GAMMA HEATING CLASSIFICATION FOR MATERIALS IRRADIATIONS

Class 1 - Active Monitors; Best Calculations

Class 2 - Passive Monitors; Good Calculations

Class 3 - Passive Monitors; Users Guide and Previous Experience

Class 4 - No Monitors; Good Calculations

Class 5 - No Monitors; Users Guide and Previous Experience

special controlled section of the FFTF core wherein constant environmental conditions are maintained. Changes external to this controlled section should have minimal effect on these long term experiments. There is little question that the actual run-to-run operation of a materials test reactor can have profound effects on materials experiments. This is particularly well illustrated by the results of experiment XX05 in EBR-II. (13) The history and experience provided by more than a decade of operating EBR-II must be incorporated in FFTF operating philosophy in order to maximize information obtained from in-core materials tests. While some experiments can no doubt be compromised by S/A interactions, an attempt should be made to hold this effect to a minimum in the controlled section of FFTF by judicious operating procedures. In analogy to EBR-II operations, these procedures are based in the main upon past experience and the demonstrated capability of calculational models.

It is, perhaps, more realistic to assume that the provision and maintenance of a controlled section in FFTF for attaining constant environmental conditions is unlikely. In general, the LMFBR environment is dynamic rather than static and evolves from a beginning of life (BOL) configuration under the influence of such factors as burn-up and structural swelling. Moreover, constant initiation of new experiments and termination of old experiments combined with the non-negligible interaction of $S / A$, which can extend not only to the nearest neighbors but even farther, creates a severe operational obstacle for such a controlled section. However, through the development of adequate calculational models, it should be possible to 
predict the effects of S/A interactions and thereby control the environment of high priority experiments within predefined tolerances. The process of developing such a capability through comparisons with experiment for the entire range of allowable reactor configurations and throughout the core, reflector and blanket regions for such parameters as fission rates, reaction rates, neutron flux-fluence spectra, gamma flux-fluence spectra, gamma heat, thermal-hydraulics, etc., comprises the overall goals of the FFTF core characterization plan.

This review focuses upon the current "state-of-the-art" of gamma heating and in-core temperature definition from the viewpoint of FFTF core characterization needs. Section II reviews the status of experimental methods used in both high and low power irradiations. Calculational methods used to analyze both high and low power experiments are considered in Section III. In Section IV, recommendations are advanced for meeting FFTF core characterization goals. A brief concluding summary can be found in Section $V$.

The preliminary nature of this treatise must be emphasized. As a consequence, no exhaustive or authoritative claims are implied. On the contrary, time does not permit citing the significant work of many who have contributed to progress in this field. In view of the pressing need to emphasize current practices at EBR-II and future requirements of FFTF, this is especially true for European efforts. The author humbly apologizes for all such oversights, which will hopefully be rectified in the future by subsequent publication of a much more comprehensive survey. 


\section{EXPERIMENTAL METHODS}

\section{A. High Power Environments}

Any discussion of gamma heating measurements in high power environments must consider both active and passive techniques. While active techniques are always more desirable since they can provide the timedependent temperature history, $T(t)$, they are not always possible due to limitations of the irradiation facility. Moreover, passive monitors can extend the utility of the irradiation facility by significantly increasing the possible number of concurrent in-core experiments. Consequently, there is considerable economic incentive to develop quality passive monitors.

\section{Active Measurements}

Active in-core temperature measurements are routinely and adequately carried out with thermocouples. Hence there is little need for a detailed exposition of thermocouple measurements here. In the temperature range of interest, $600-1400^{\circ} \mathrm{F}$, Chromel-Alumel thermocouples work reliably and provide very good accuracy. Uncertainties of at most a few degrees (F) are representative of calibrated in-core temperature measurements with these thermocouples. It is important to recognize that this high accuracy cannot be maintained for temperatures much above the region of interest here, due primarily to electrical shunting effects in insulating materials. It is interesting to note in passing that a program has been underway at NRTS for some time to develop an ultrasonic thermometer ${ }^{(14)}$ which would be applicable up to roughly $3000^{\circ} \mathrm{C}$ and hence be of interest in certain fuel experiments. The present capability of this ultrasonic thermometer now extends up to $2700^{\circ} \mathrm{C}$ with accuracy ranging from $\pm 50^{\circ} \mathrm{C}$ to $\pm 100^{\circ} \mathrm{C}$. (15)

In-core gamma ray heating can be actively measured with calorimeters, which in turn require active temperature monitors. Consequently the established capability of accurate in-core temperature measurements with Chromel-Alumel thermocouples has been used to advantage in the design and subsequent development of in-core calorimeters to measure gamma heating. The current "state-of-the-art" of gamma-ray heat calorimetry is well represented by the experiments of Koenig and co-workers at EBR-II. (16) 
The design of this calorimeter is shown in Figure 1, where the internal (gamma plus neutron) heat generation $Q$ is related to the temperature $T$ at axial location $x$, by

$$
Q=(k / \rho)\left(T-T_{c}\right)\left(L x-x^{2} / 2\right)
$$

In Equation (1) $k$ is the thermal conductivity, $\rho$ is the density, $T_{c}$ is the cold end temperature, and $L$ is the total susceptor length. (The axial position $x$ is the distance between the cold end and the location of the thermocouple). A consistent set of units for Equation (1) is: $T$ and $T_{c}$ in ${ }^{\circ} \mathrm{F}, X$ and $L$ in $\mathrm{cm}, \rho$ in $\mathrm{gm} / \mathrm{cm}^{3}, Q$ in watts $/ \mathrm{gm}$, and $k$ in watts $/\left({ }^{\circ} \mathrm{F} \cdot \mathrm{cm}^{2}\right)$.

Vacuum is maintained in the annulus between the susceptor and outer wall, 0.1 torr, to render negligible any radial heat transfer by conduction. Radial heat loss by radiation must be taken into account. In the initial efforts with these calorimeters, it was the inadequate treatment of radiative heat transfer which produced discrepancies between theory and experiment; moreover early theoretical efforts were also subject to certain discrepancies which have subsequently been amended. The resulting agreement improved in a manner normally anticipated by the proper application of the scientific method, i.e., continuing trial and error improvement by uncovering systematic effects either by examination of theory and experiment independently or through comparison of calculations and measurements.

The overall experimental error of these EBR-II calorimetry measurements has been reduced to about $7 \%$ and is presently dominated by uncertainties in the thermal conductivity $k$ and emissivity $\varepsilon$ of stainless steel. Improved accuracy could be afforded future gamma heat calorimetry by:

a) Thermocouple measurements of cold-end temperature, $T_{c}$.

b) Use of coated components (susceptor and outer wall) or the introduction of a concentric cylinder in the vacuum region to serve as a radiation shield.

c) Construction of calorimeters from materials other than stainless steel. 


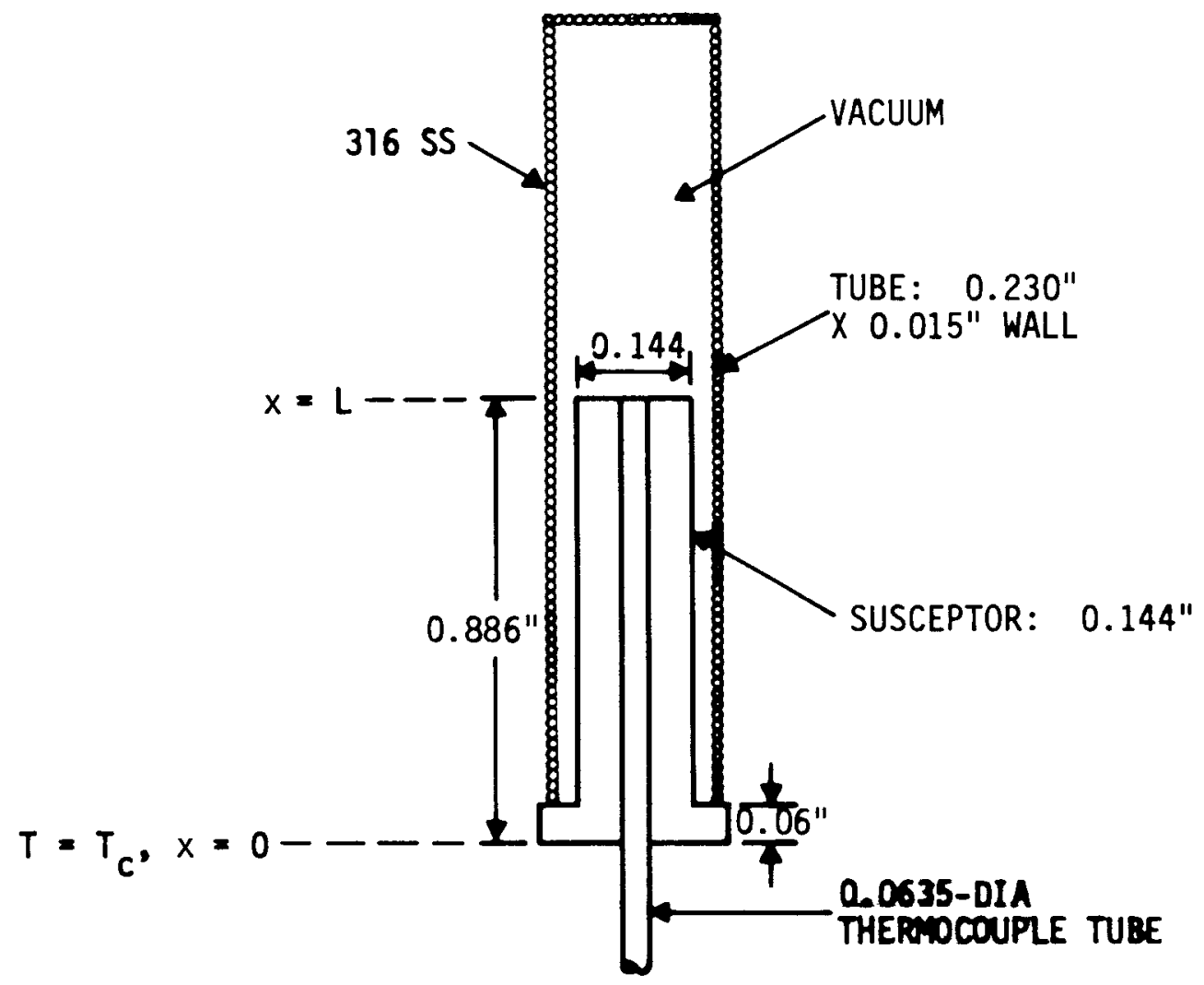

HEDL $7511-67.4$

FIGURE 1. Calorimeter in Current Use at EBR-II for Active Measurement of Gamma (and Neutron) Heating. 
The cold-end heat sink temperature $T_{c}$ is usually inferred from either the local coolant temperature of some nearby thermocouple (or some combination of both). Hence $T_{c}$ is not known as accurately as $T$, a fault which can easily be remedied by introducing a second thermocouple in the calorimeter. Radiative heat losses can be dramatically reduced by using coated components or introducing a concentric cylindrical heat shield. Coatings such as gold, silver, nickel, aluminum ... etc. can be considered. For high fluence in-core irradiations, the surface instability of gold-coated components may introduce undesirable effects and therefore warrants investigation. As an alternative, it may be necessary to coat calorimeter components with other materials in order to avoid surface instability effects. The use of coated components appears to be more advantageous, than use of a heat shield, since it maintains the simple (original) geometry and can provide a well known surface emissivity. (17) Both factors should permit more accurate estimates of radial heat loss by radiation. Further improvement is possible by selection of calorimetry materials for which the thermal conductivity is well known, since that of stainless steel is rather poorly known. (18) By incorporating these simple modifications, gamma heat calorimetry of better than $5 \%$ and perhaps as low as $3 \%$ should be possible.

Construction of calorimeters using given materials can, on the other hand, be justified by sufficient interest in total heating (gamma plus neutron plus $\beta$-decay) in specific types of materials (e.g. SS 304 , SS $316, \ldots$ etc.). The choice of alternate calorimeter materials for accurate gamma-heating measurements is, however, limited and should meet the condition that $\beta$-decay heat generation (through neutron activation) represent a negligible perturbation to internal (gamma plus neutron) induced heat generation. From the viewpoint of calculations, a strong motivation for introducing different calorimetry materials independently exists and will be discussed in Section IV.A.1.

Other significant reactor calorimetry efforts have existed, $(19,20)$ which demonstrate that successful measurements are possible over an enormous range of materials and elements extending from $Z=4(B e)$ up to $Z=83(B i)$. 
In contrast with steady-state calorimetry employed in EBR-II, in-core reactor calorimetry can also be based upon observations of the time derivative $\mathrm{dT} / \mathrm{dt}$. In this latter technique, the time rate of change of temperature, $d T / d t$, is observed immediately after calorimeter insertion into (or withdrawal out of) a reactor (that is already operating at a constant power leve1). This technique has been independently advanced in high power reactors ${ }^{(20)}$ and low power critical experiments. ${ }^{(21)}$ The need to insert (or withdraw) the calorimeter completely into (or out of) the core environment with the reactor at power renders this technique inapplicable for many high power reactors such as EBR-II and FFTF. However, the sensitivity of this calorimetry method for special low power calibration experiments should not be overlooked.

\section{Passive Measurements}

Many concepts have been proposed for passive in-core temperature monitoring. Ideally, one would like a passive monitor to provide the entire temperature history, $T(t)$, of a given in-core exposure. It would also be desirable to carry out postirradiation measurements with considerably greater ease than is customarily attendent in hot-cell type measurements. As has already been indicated, due to limited interest and support only a few of these proposed passive techniques have been investigated with any degree of rigor. Failure to pursue any single one of these concepts cannot therefore be construed as evidence of elimination through systematic study and evaluation. In this perspective, one cannot be certain that historical conclusions are necessarily correct. Indeed, further study of any of these discarded and/or unpursued concepts could provide unexpected surprises.

Melt wires, perhaps the earliest passive temperature monitor, provide discrete range temperature estimates according to the melting points of the various metals and alloys used. The disadvantage of noncontinuous monitoring is often highlighted by failure to establish a temperature range for an in-core exposure, in which event only an upper or lower temperature bound is determined. In addition, hot cell radiography is required to examine 
the physical state of the melt wires. Not only is the process cumbersome, but in many instances difficult to quantify. Here, melting can only be indicated through either change of shape or loss of image definition in the radiograph. Consequently, because of very limited accuracy and postirradiation difficulties, melt wires are no longer in normal use.

Another proposed passive monitor, silicon carbide, has similarly fallen into disuse. Two different properties of $\mathrm{SiC}$ have been proposed and used for passive in-core temperature monitoring, namely postirradiation length change or lattice parameter recovery by annealing. It has been advanced that annealing of irradiation induced elongation in SiC depends linearly on the difference between annealing and irradiation temperatures, with little or no recovery occurring for annealing temperatures below the irradiation temperature. The variation of the lattice parameter in SiC is thought to saturate at a fluence of roughly $3 \times 10^{20} \mathrm{n} / \mathrm{cm}^{2}$, with the saturation value dependent upon irradiation temperature. Both of these SiC properties were investigated for passive in-core temperature monitoring in EBR-II and both properties were generally found wanting in reliability and accuracy. (22) As a result of this appraisal, SiC has not been actively used or seriously considered for further systematic study.

More speculative concepts have been advanced, which are based upon structure related properties that depend simultaneously upon both fluence and temperature. This class of passive monitors would include such properties as void production, Frank loops, dislocations, etc., where in size and/or number density depend on fluence and temperature. While these properties can be routinely used by experienced observers for qualitative temperature estimates, little if any quantitative basis has been established through even preliminary investigations of these possibilities. Included in this class of passive monitors is irradiation produced gas, e.g., helium production in stainless steel, wherein postirradiation gas release would depend not only upon fluence but annealing temperature as well. Here the existence of temperature dependent "traps" has been postulated as a mechanism for retention of the gas until the annealing temperature exceeded the activation temperature corresponding to a given "trap", whereupon a 
significant increase in gas release should occur. Cursory examination of this concept has been conducted for helium produced in stainless steel irradiations with, unfortunately, little if any support for the "trap" postulate of occluded gas.

Still another class of passive monitors which has been proposed is based upon phase transitions in suitably chosen materials, wherein some observed property of the phase transition possesses the desired temperature dependence. Melt wires are perhaps the simplest form of passive monitor in this class. Another passive monitor in this class is the vapor pressure monitor or VAP, ${ }^{(23)}$ which utilizes vapor pressure of certain elements as the temperature dependent variable. A possible in-core VAP design is shown in Figure 2. At a given (in-core) temperature $T$, the quartz VAP chamber will contain an equilibrium vapor pressure of source material, which depends strongly upon $T$. Upon return to room temperature, $T_{R}$, source material will be precipitated on the chamber wall. The amount of precipitated source material (per unit area) is related to $\left(T-T_{R}\right.$ ) and upon suitable calibration provides the desired temperature dependent variable. In spite of apparent advantages, the VAP concept has not been systematically evaluated or used.

Perhaps the most simple, reliable, and accurate passive monitor for measuring temperature of in-core irradiations is the Thermal Expansion Difference (TED) monitor. TED monitors are relatively new ${ }^{(24)}$ and have benefitted from more systematic development than most other proposed schemes. (25) Figure 3 displays the current TED design, which essentially is a Type 304 stainless steel cylinder filled to capacity with sodium and then sealed by appropriate welding techniques. This method is based on the difference in temperature-dependent expansion between sodium and stainless steel. At elevated temperature, the sodium expands more rapidly than the stainless steel creating plastic deformation which can be measured after the removal of the TED from the in-core reactor environment. Current practice is to use immersion density methods to measure TED deformation. Out-of-core calibrations provide an accurate empirical relation between immersion density change and temperature. TED monitors respond to the maximum temperature encountered during the irradiation. 


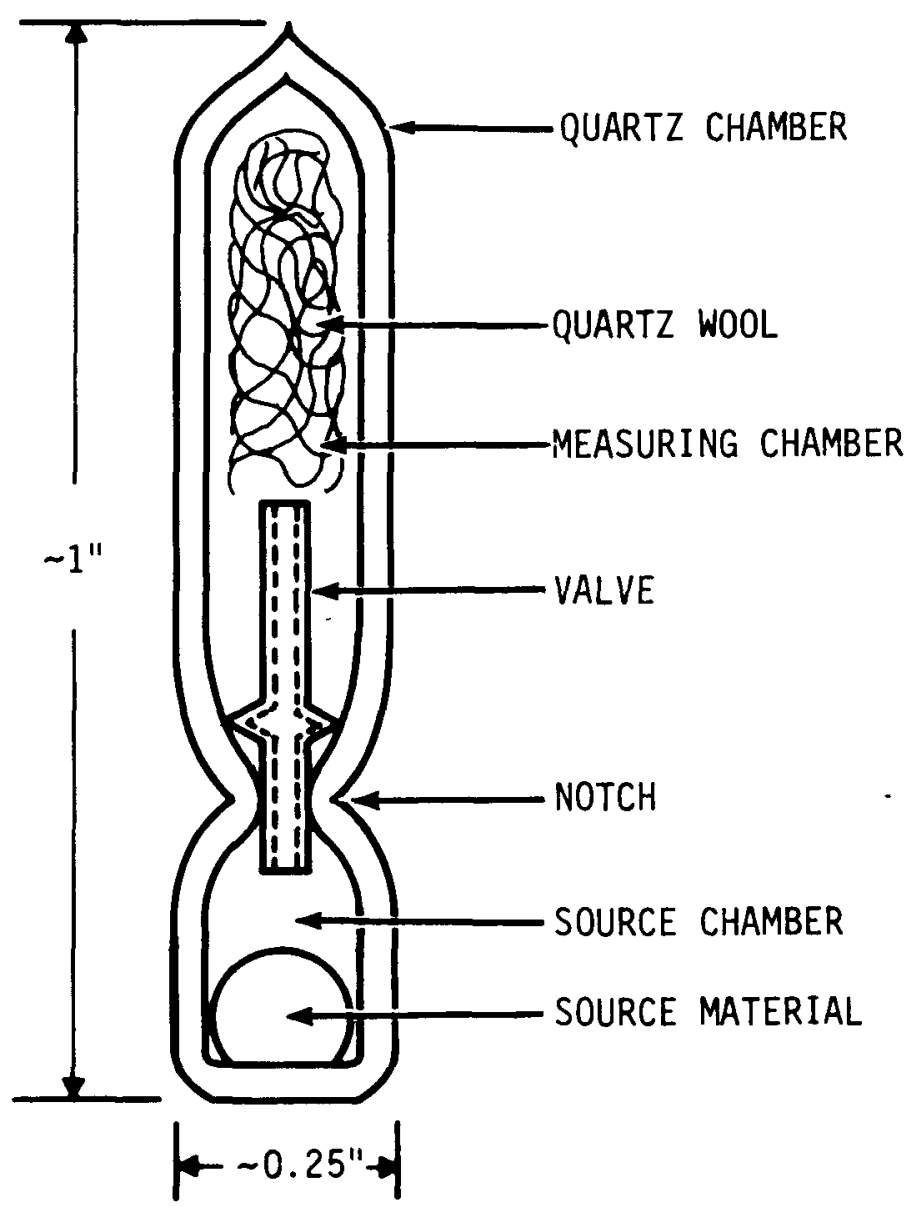

HEDL 7511-67.5

FIGURE 2. Design of Passive Vapor Pressure (VAP) Monitor for In-Core Temperature Measurements. 


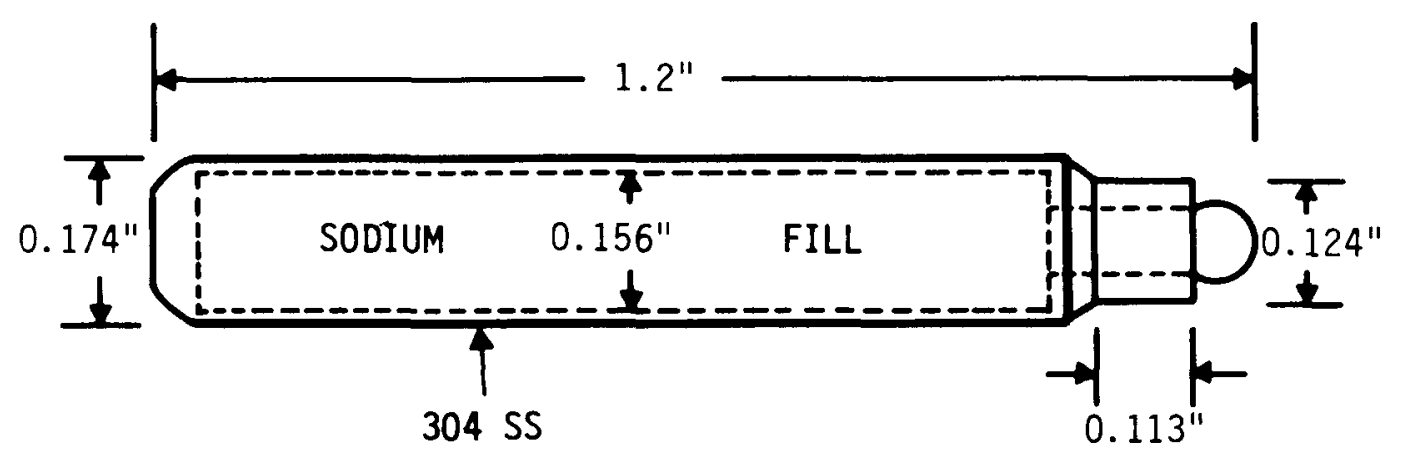

HEDL 7511-67.3

FIGURE 3. Passive Thermal Expansion Difference (TED) Monitor in Current Use at EBR-II. 
The major limitation of TED monitors is radiation induced swelling which can contribute a non-zero component to observed immersion density change for high fluence exposures. The reported $(25,26)$ accuracy of TED monitors ranges from $\pm 5^{\circ} \mathrm{F}$ to $\pm 10^{\circ} \mathrm{F}$, al though actual TED measurements reveal a current in-core reproducibility which can be somewhat larger and extends up to $\pm 20^{\circ} \mathrm{F}$ or higher. (27) However, these latter measurements were obtained at a fluence close to $10^{22}$, which is thought to be in the region where TED correction for radiation induced swelling would be necessary. In fact, recent TED measurements in EBR-II instrumented S/A XX07 ${ }^{(28)}$ reveals that this effect is considerable at a fluence of approximately $3.4 \times 10^{22}$. In this experiment, along with ordinary TED monitors, "dummy" TED monitors filled with helium instead of sodium, were simultaneously irradiated. The "dummy" TED monitors were expected to provide a basis for correction of radiation induced swelling. Comparison of corrected TED results with thermocouple observations in $\mathrm{XX07}$ is awaiting more detailed data analysis. Nonetheless, it is evident that development of TED monitors using swelling resistant materials such as Inconel 600 or 625, instead of the currently employed stainless steel 304, must be actively pursued. To this end, al ternate materials have been considered at the EBR-II project. (29)

An interesting outgrowth of TED monitors is a passive gamma heat calorimeter called the Gamma Expansion Difference Monitor (GEDM). (25) The design of the GEDM is shown in Figure 4, wherein the void formed between the susceptor and inner capsule is filled with sodium, which therefore comprises essentially a TED. The (maximum) gamma heat generation rate in the susceptor is determined from the (maximum) temperature measured by the TED and the known heat transfer characteristics of the GEDM configuration. Actual evaluation of the GEDM concept must await initial in-core tests, which are presently underway in EBR-II. ${ }^{(30)}$ One attractive feature of the GEDM would be the ability to fabricate passive gamma heat calorimeters from different materials, an advantage which has already been cited for active gamma heat calorimetry.*

*See Section IV.A. 1 in this regard. 


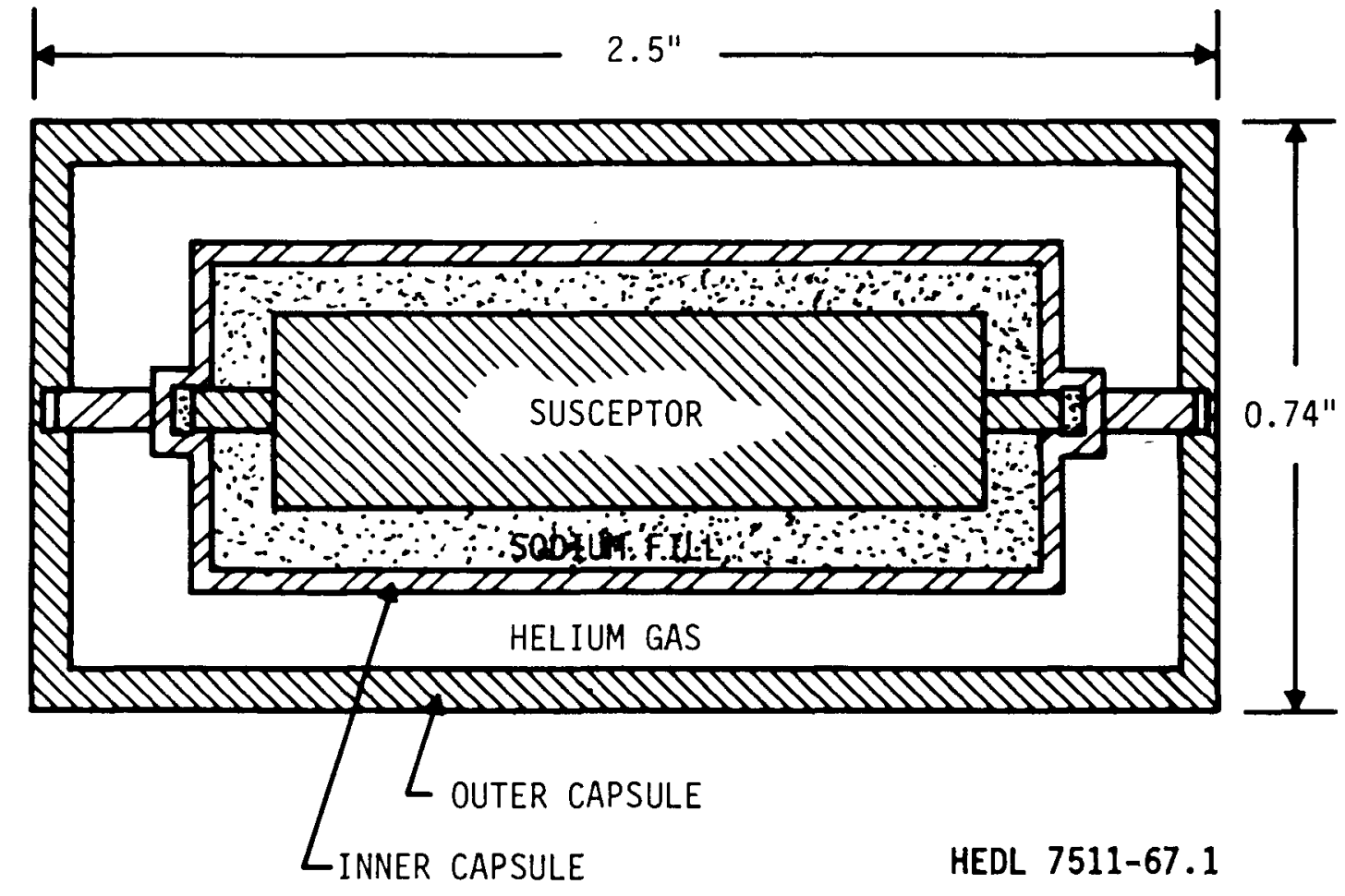

FIGURE 4. Passive Gamma Expansion Difference (GEDM) Monitor Under Test at EBR-II. 
The selected passive monitors described above are compared qualitatively in Table IV. While one would ideally like to determine the complete timedependent temperature history $T(t)$ of any given irradiation, unfortunately none of these passive monitors is capable of this task. All of these passive monitors provide, instead, the maximum temperature of the irradiation. In addition to falling short of ideal goals, this "state-of-the-art" limitation can create serious difficulties and misinterpretations, such as those recently encountered in EBR-II experiments. In this example, passive monitors (principally TEDs) located well below reactor mid-plane yielded results consistently higher than comparable active measurements. Moreover, experienced workers could not reconcile these higher temperature values with other observations recorded on their irradiated specimens. These anomalies were resolved eventually with the postulation and confirmation of a "thermal plume" formation above the core during the cover lift operation after reactor shutdown. (27) The work of C. C. Price and J. L. Gillette of the EBR-II Project has verified that temperatures from $800^{\circ} \mathrm{F}$ up to $840^{\circ} \mathrm{F}$ can exist in this "thermal plume" region above the core. Consequently passive monitors which would normally register in-core temperatures below "thermal plume" values will be compromised, since each S/A must be inserted and/or removed through this "thermal plume" region. Since it is possible that comparable effects could arise for passive monitors in FFTF, an attempt to identify any FFTF operation analogous to the cover lift operation in EBR-II should be initiated with the hope that any such FFTF operation can still be suitably modified or circumvented.

TABLE IV

QUALITATIVE COMPARISON OF PASSIVE TEMPERATURE MONITORS

\begin{tabular}{|c|c|c|c|c|}
\hline Monitors & $\begin{array}{c}\text { Post Irradiation } \\
\text { Difficulties }\end{array}$ & Reliability & Accuracy ${ }^{a}$ & $\begin{array}{c}\text { Fluence } \\
\text { Sensitivity }\end{array}$ \\
\hline Melt Wires & High & Fair & $30-100^{\circ} \mathrm{F}$ & None \\
\hline SiC & High & Fair & $\sim 100^{\circ} \mathrm{F}$ & Low to Moderate \\
\hline VAP & Moderate & Untested & Untested & None to Low \\
\hline TED & Moderate & High & $10-20^{\circ} \mathrm{F}$ & Moderate \\
\hline
\end{tabular}




\section{B. Low Power Environments}

One of the primary testing beds for fast reactor neutronics calculations has been low power experiments conducted in fast critical facilities both here and abroad. Many experiments of interest cannot be carried out in high power reactors either through limitations of space and operating time or due to the nonexistence of suitable experimental techniques. The adaptation of high power reactors, such as EBR-II, for particular experiments is either impossible or impractical. For such cases, it is of considerable economic benefit to employ special fast critical assemblies which afford proper access, environment, and overall irradiation conditions at low power that are more conducive to the generation of accurate experimental results.

The inapplicability of many experimental techniques in high power environs can be traced to inherent limitations. A classification of inherent high power limitations has already been given for neutron measurements, ${ }^{(5)}$ which is also applicable for gamma-ray measurements. As cited in this classification, the principal reasons for high power inapplicability are:

1) count rate limitations,

2) radiation damage (of detectors and/or electronic components),

3) Sensitivity to background radiation components, and

4) temperature sensitivity (of detectors and/or electronic components).

As discussed in the introduction, attention has almost been entirely focused on measurements of the neutron component, to the practical exclusion of other important considerations, such as measurement of the gamma-ray component of in-core radiation fields. This is, indeed, unfortunate, since special fast critical facilities have existed and operated for many years, which could have been more fully exploited were low power gamma-ray experiments properly incorporated into existing programs. In analogy to neutron measurements in low power fast critical assemblies, both integral and differential measurements of the gamma-ray component are possible. Gammaray dosimetry measurements are analogous to integral neutron activation type 
measurements, whereas gamma-ray spectrometry possesses a direct analogue in neutron spectrometry. Consequently, the present assessment of low power gamma-ray measurements falls quite naturally into a consideration of integral-dosimetry measurements and differential-spectrometry measurements.

\section{Gamma-Ray Dosimetry}

The earliest measurements of gamma dose carried out in low power critical assemblies utilized film badges, in much the same manner as film badges have been employed for years in personnel dosimeters. Much can be said of the film badge technique, which is simple and has been well exploited since the very dawn of radiation measurements. In particular, film badges provide permanent records, which therefore permit storage for re-examination or analysis at any future time deemed necessary. In addition, film badges possess very low sensitivity to fast neutrons and hence are appropriate for gamma dose measurements in fast critical assemblies.

Perhaps the simplicity and automation of readout is the main reason Thermoluminescent Dosimetry (TLD) has supplanted the film badge technique for both personnel dosimetry and low power critical assembly gamma dosimetry measurements. In the last decade, considerable study has been expended on understanding and applying the TLD method of measuring gamma dose. (3I) In this method, ionizing radiation creates free electrons or holes in special Thermoluminescent (TL) materials, which possess traps that are stable at room temperature. After irradiation, the TL material is heated and the electrons or holes are released by thermal excitation. Thermoluminescent emission occurs through electron-hole annihilation at recombination centers. The intensity of the TL emission (usually observed in the visable light region with photocells or multiplier phototubes) is related to the exposure dose. While the TLD method can be used up to very high dose $\left(\sim 10^{4} R\right)$, it should be apparent that this method cannot work at the high temperatures found in power reactors and an upper temperature bound of somewhat less than $100^{\circ} \mathrm{C}$ is advisable.

The first TLD measurements is U.S. low power critical facilities were carried out to measure gamma heating in a thermal mockup of the Argonne 
Advanced Research Reactor $\left(A^{2} R^{2}\right),(32)$ whereas the ZEBRA fast critical facilities were used in the UK. (33) Serious efforts to measure gamma heating were not really initiated until the early 1970's, when concern arose with regard to fast reactor shielding $(34,35)$ and gamma heating in control rods. (36) Prior to that time, proper support was not provided and measurements lacked a quantitative base. It is, indeed, fortunate that since that time an in-depth systematic study of the applicability of TLD methods in fast critical assemblies has taken place. (37)

The results of these efforts reveal that the TLD method must be applied with utmost care and attention to detail, otherwise precision and accuracy of gamma dose measurements are easily compromised. Uncertainties in TLD measurements have been reduced by careful and systematic examination of every aspect of the experimental technique. In this manner, a "state-ofthe-art" precision approaching $\pm 3 \%$ has been attained. A limited discussion of the experimental technique required to attain this level of precision is therefore warranted.

Although two TL materials are available, CaF and LiF, the best results have been obtained with small ${ }^{7} \mathrm{LiF}$ rods, depleted in the ${ }^{6} \mathrm{Li}$ isotope so as to reduce neutron sensitivity. The $\mathrm{CaF}_{2}: \mathrm{Mn}$ TLD is very sensitive to visible light and difficult to read out. Although TLDs are manufactured by the hundreds (as required in critical assembly experiments), they are not produced with the quality control demanded in high precision measurements. Considerable batch-to-batch variation in sensitivity exists, which makes it imperative to use only ${ }^{7}$ Lif TLDs from a single batch in a given in-core experiment. Even then, sensitivity variations can reach $20 \%$ or higher within a single batch. Consequently, all TLDs in a given batch are intercompared by irradiation in the same gamma field, where upon specimens which do not 1 ie within $\pm 5 \%$ of the mean value are discarded. In addition, this sensitivity preselection process provides the remaining TLDs with normalization factors that are used for subsequent in-core exposures. In this manner, all TLDs that are retained possess a normalized sensitivity equivalent to the batch mean. 
One hundred or more TLDs are necessary for accurate calibration of the TLD readout system. Calibrations are normally conducted with a ${ }^{60} \mathrm{Co}$ source of (accurately) known source strength and these irradiations provide an empirical relation between TLD light output (usually measured in nanocoulombs) and exposure dose. It has been shown that TLD light output can vary substantially, by up to a factor of two or more, with different preannealing and postannealing techniques. Hence, strict reproducibility of preannealing, postannealing and read-out procedures must be maintained, otherwise precision will be sacrificed and gamma dosimetry experiments will become beset with erratic data (as was typical of early TLD observations). For work at the highest precision achieved to date, $\pm 3 \%$, it is not advisable to reuse TLDs after in-core irradiations, where exposure to fast neutrons can affect sensitivity for subsequent measurements.

Accuracy available from TLD observations depends not only upon precision and absolute calibration, but corrections for neutron sensitivity and gamma spectral sensitivity of the detector. Correction for neutron sensitivity depends upon neutron spectrum and ranges up to roughly $12 \%$ in hard LMFBR type neutron spectra. Moreover, this correction is based on very early ${ }^{7}$ LiF response data, ${ }^{(38)}$ which can only be indirectly inferred as applicable to current experiments. Overall TLD sensitivity depends crucially upon the specific type, manufacture and commercial supply of

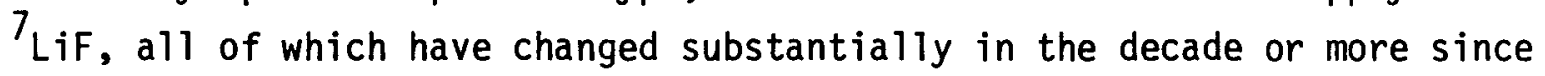
these early TLD sensitivity studies. This is a current deficiency which must be amended. Accurate sensitivity experiments must be repeated with

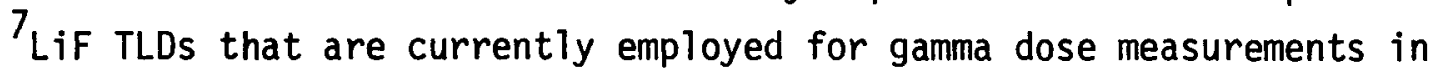
critical assemblies.

For dosimetry measurements, the TLD is fully enclosed by material for which the actual gamma heat generation measurement is desired, e.g., stainless steel is often of interest. The enclosing capsule must be thick enough to insure establishment of electronic equilibrium. Since the surrounding medium and dosimeter are of different composition, the energy absorbed per unit mass in the medium ${ }_{m} E_{M}$ differs from the energy absorbed 
per unit mass in the dosimeter $\mathrm{m}_{\mathrm{D}}$. Extensive analytical work conducted over the years, which has become known as Cavity-Ionization theory ${ }^{(39)}$ relates these doses in the form

$$
m_{M}=\left(\frac{l}{f}\right) \cdot m^{E_{D}}
$$

where the correction factor $f$ is generally a function of gamma-ray energy, dosimeter size, and the composition of the dosimeter and surrounding medium. If follows that correction for gamma-ray spectral sensitivity lies in the different TLD response, to empirical calibrations using monoenergetic gamma-rays (whether ${ }^{60}$ Co or other strong sources) and to subsequent exposure in completely different in-core gamma-ray continua.

For a given in-core exposure, the appropriate correction factor can be obtained from a suitable average of $f^{-1}$ over the gamma continuum experienced by the TLD. The gamma-ray energy dependence of $f^{-1}$ for a ${ }^{7}$ iF TLD and encapsulating materials of interest, such as iron, tantalum, and $\mathrm{B}_{4} \mathrm{C}$, has been calculated for the energy region from 0.03 to $10 \mathrm{MeV}$. (37) The $f^{-1}$ correction factor can vary from unity by one to two orders of magnitude. The deviation from unity is only small in the limit of high energy gamma-rays (i.e., when small-cavity ionization theory would be applicable) and rapidly becomes significant with decreasing energy. This correction is enormously sensitive to the degree of mismatch between the dosimeter and medium composition (i.e., atomic number $Z$ ) and reduces to tractable proportions only when these materials are well matched.

This dependence is clearly less than desirable for two obvious reasons:

1) Substantial error can be incurred when $f^{-1}$ deviates far from unity.

2) TLD inferred dosimetry data are dependent upon gamma-ray exposure spectra.

In practice, the spectral averaged correction factor $\left\langle f^{-1}\right\rangle$ is obtained from calculated gamma spectra. As a consequence, TLD inferred doses will 
not be independent of calculation. This dependence is very weak for $\left\langle\mathrm{f}^{-1}\right\rangle$ close to unity, in which event the error entailed by this correction also becomes manageable. Under such ideal conditions, which have been fortunately verified for stainless steel - ' LiF TLD's in fast critical facilities, $(40)$ the overall accuracy is estimated at approximately $10 \%$ and is principally comprised of uncertainties in:

1) TLD precision

2) Absolute calibration

3) Correction factor for neutron sensitivity

4) Correction factor for gamma-ray spectral sensitivity.

2. Gamma-Ray Spectrometry

As a result of formidable experimental difficulties compounded by lack of support, in-core measurements of gamma-ray spectra have been practically nonexistent. This serious deficiency was recognized independently some ten years ago by Silk in the UK ${ }^{(41)}$ and Gold in the U.S., (42) who not surprisingly pursued the development and application of the very same experimental method, namely Compton Recoil Gamma-Ray Spectroscopy. In this method of gamma-ray spectrometry (which is analogous to in-core proton recoil methods of fast neutron spectrometry), one records the electron recoil continuum induced in a suitable detector by the in-core gamma spectrum. It was the advent of large lithium drifted silicon solid state detectors during this time period which made this method a practical reality. Small in-core probes of adequate sensitivity could be fabricated using these large $\left(\sim 1 \mathrm{~cm}^{3}\right) \mathrm{Si}(\mathrm{Li})$ detectors. Moreover, the fast neutron sensitivity of $\mathrm{Si}(\mathrm{L} i)$ detectors is low relative to the gamma response and therefore does not present the insurmountable problems normally encountered with other types of gamma detectors, such as NaI scintillators.

Using $\mathrm{Si}(\mathrm{Li})$, as opposed to $\mathrm{Ge}(\mathrm{Li})$, solid-state gamma detectors not only simplify in-core experiments, but permit accurate description of the detector response kernel with the Klein-Nishina formula for Compton scattering. In low $Z$ media such as silicon, Compton scattering dominates 
all other interactions by more than two orders of magnitude over a broad energy region of interest for in-core gamma spectrometry. Consequently the gamma ray spectrum can be obtained from an unfolding calculation, using the electron continuum measurements of $\mathrm{Si}(\mathrm{Li})$ detectors together with a response kernel accurately defined ( $2 \%$ uncertainty) by the Klein-Nishina formula. Although this method has been fruitfully applied in both health physics $(43)$ and environmental science, $(44,45)$ it is quite unfortunate that initial efforts in reactor applications have remained relatively unexploited and unpursued.

As a consequence, in-core gamma-ray spectromeiry utilizing Compton Recoil Gamma Ray Spectroscopy has neither evolved nor improved in the time period ( 25 years) since last reviewed. (46) Hence, any summary of the "state-of-the-art" for in-core gamma spectrometry need only consider improvements and developments up to that time. Absolute measurements by Compton Recoil Gamma Ray Spectroscopy are possible in the energy 0.2-2.2 MeV with an accuracy of approximately 20\%. Experimental error is dominated by correction for finite detector size [i.e. electron escape from the $\mathrm{Si}(\mathrm{Li})$ detector] and uncertainty in the total number of electrons in the sensitive volume of the detector. The upper energy limit of applicability, 2.2 $\mathrm{MeV}$, arises through electron escape from the $\sim 1 \mathrm{~cm}^{3} \mathrm{Si}(\mathrm{Li})$ detector. In spite of the ability to correct for finite size effects, counting statistics become unacceptably low for observed events in the pulse height region beyond $2 \mathrm{MeV}$. The lower energy bound of applicability is created by the rapid increase in photoelectric effect in silicon with decreasing gamma-ray energy below $0.2 \mathrm{MeV}$. Fast neutron induced events, principally due to elastic scattering, also contribute to the low pulse height (energy) end of the observed electron continuum. Both of these effects combine to define a lower limit of applicability, which has been conservatively set at $\sim 0.2 \mathrm{MeV}$.

The capability of Compton Recoil Gamma-Ray Spectroscopy is demonstrated in Figure 5. This observation was carried out in ZPR-6, Assembly No. $6{ }^{(46)}$ and to the author's knowledge is the only in-core gamma spectrum measurement ever conducted in a fast reactor environment. The structure and 


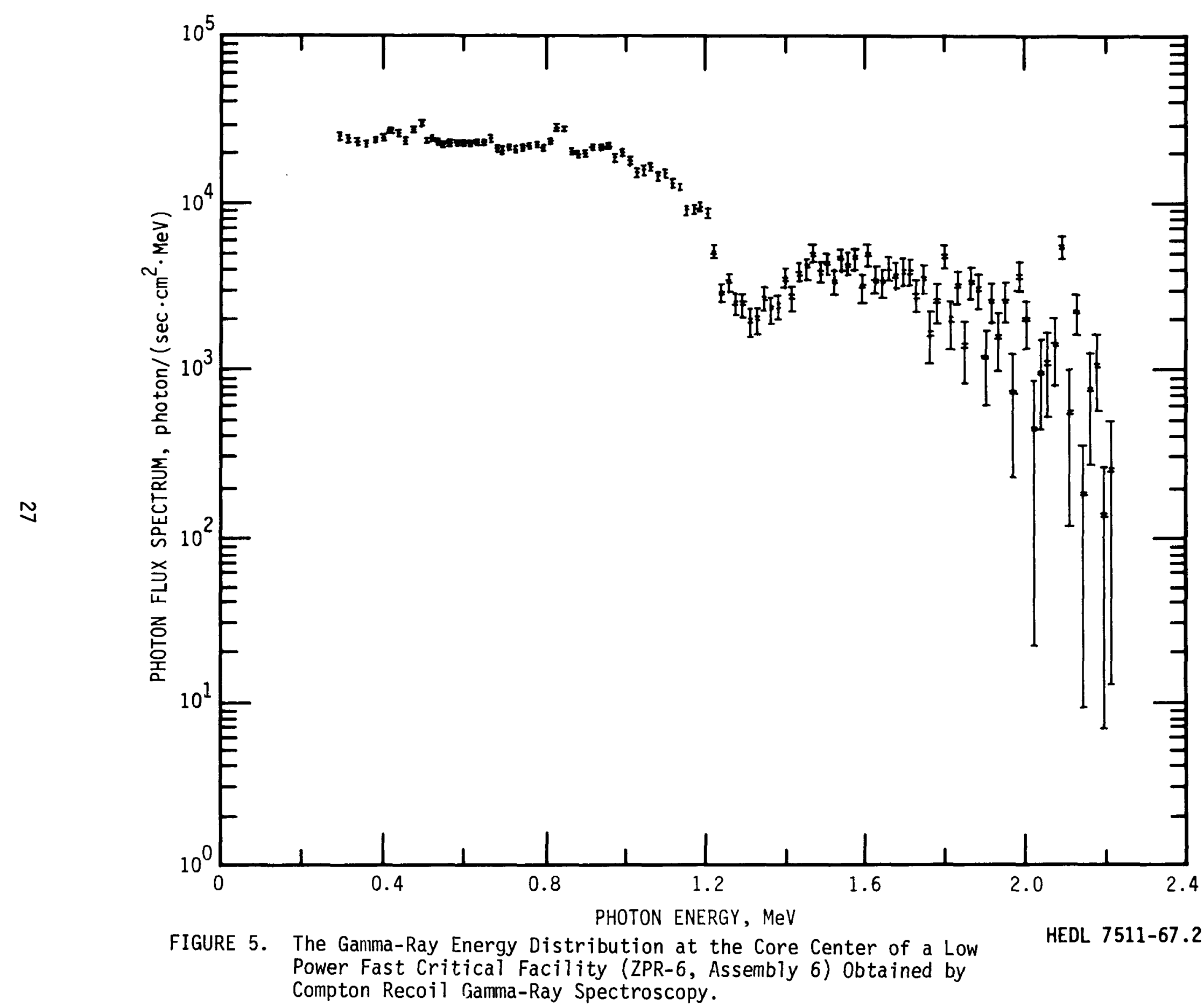


spectral detail evident in this spectrum is afforded by the good electron energy resolution, $210 \mathrm{KeV}$ (FWHM), obtained with an in-core Si(Li) detector cooled to roughly $-20^{\circ} \mathrm{C}$. For example, the three peaks in this spectrum near $0.85,1.3$, and $2.1 \mathrm{MeV}$ correspond to excitation of levels in ${ }^{56} \mathrm{Fe}$ produced by fast neutron in-elastic scattering. (Iron is one of the dominant constituents of the central core region in ZPR-6, Assembly No. 6). While room temperature operation of the $\mathrm{Si}(L i)$ detector is possible, resolution would be seriously compromised and much spectral detail would be lost. Operation at much above room temperature is precluded through vastly increased solid-state detector noise. Measurements carried out at $-20^{\circ} \mathrm{C}$, such as depicted in Figure 5, offer much finer spectral detail than current calculational models, which possess relatively broad group structure (see Section III).

A particularly important outgrowth of Compton Recoil Gamma-Ray Spectroscopy is a method of absolute gamma-ray dosimetry $(47,48)$ which does not require the use of other detectors or other radiation standards. Absolute gamma-ray dosimetry by recoil electron spectroscopy currently possesses an accuracy of roughly $10 \%$ for low $Z$ media, independent of gamma spectrum. Hence, it can be applied in fast critical facility experiments to assess the accuracy of any other given integral dosimetry technique, especially under conditions of significantly changing gamma spectra. Since rapid spectral changes are to be anticipated in fast reactor environments, this method can be applied for independent verification and/or improvement of the accuracy of different in-core integral gamma dosimetry techniques. In view of the increasing application of TLD in fast critical assemblies, comparison with this method would be particularly productive. 
III. CALCULATIONS

Serious analytical efforts for calculating gamma heating effects in LMFBR environments have been launched only within the last five years or so. As pointed out in the introduction, these efforts were motivated by a number of independent concerns. Perhaps the most important of these are:

a) Transition of EBR-II from a demonstration plant to an irradiation facility.

b) Evaluation and comparison of potential LMFBR control rod materials.

c) Evaluation of safety-related phenomena, such as gamma heating in structura 1 components and cooling requirements for after shutdown decay heat.

d) LMFBR shielding.

Both a) and b) provide the underlying motivation for calculational efforts carried out at Argonne National Laboratory (ANL), whereas b), c) and d) are responsible for parallel work at the Hanford Engineering Development Laboratory (HEDL). At ANL, calculations have been conducted by the EBR-II project for gamma heating characterization at high power, where comparison with in-core calorimetry is possible (see Section II.A.1). In addition, calculations were also carried out by ZPR-ANL personnel for analysis of control rod experiments using the low power fast critical ZPPR facility, where comparisons could be made with TLD measurements (see Section II.B.1). Calculations at HEDL have also been directed to the analysis of special low power critical experiments both for control rod studies and FFTF shielding requirements, both of which employed TLD gamma dose measurements. In the latter instance, the FFTF was simulated using the Engineering Mock-Up Critical (EMC) at ZPR-9.

The overall approach used for calculating gamma spectra and related phenomena is generally the same in all of these efforts. The block diagram given in Figure 6 is a graphical presentation of this overall approach. Substantial differences in detail do exist, however, for the ANL and HEDL computations, whereas the detailed work conducted by the EBR-II project and ZPR-ANL personnel are quite similar. This similarity reflects, no doubt, the close 


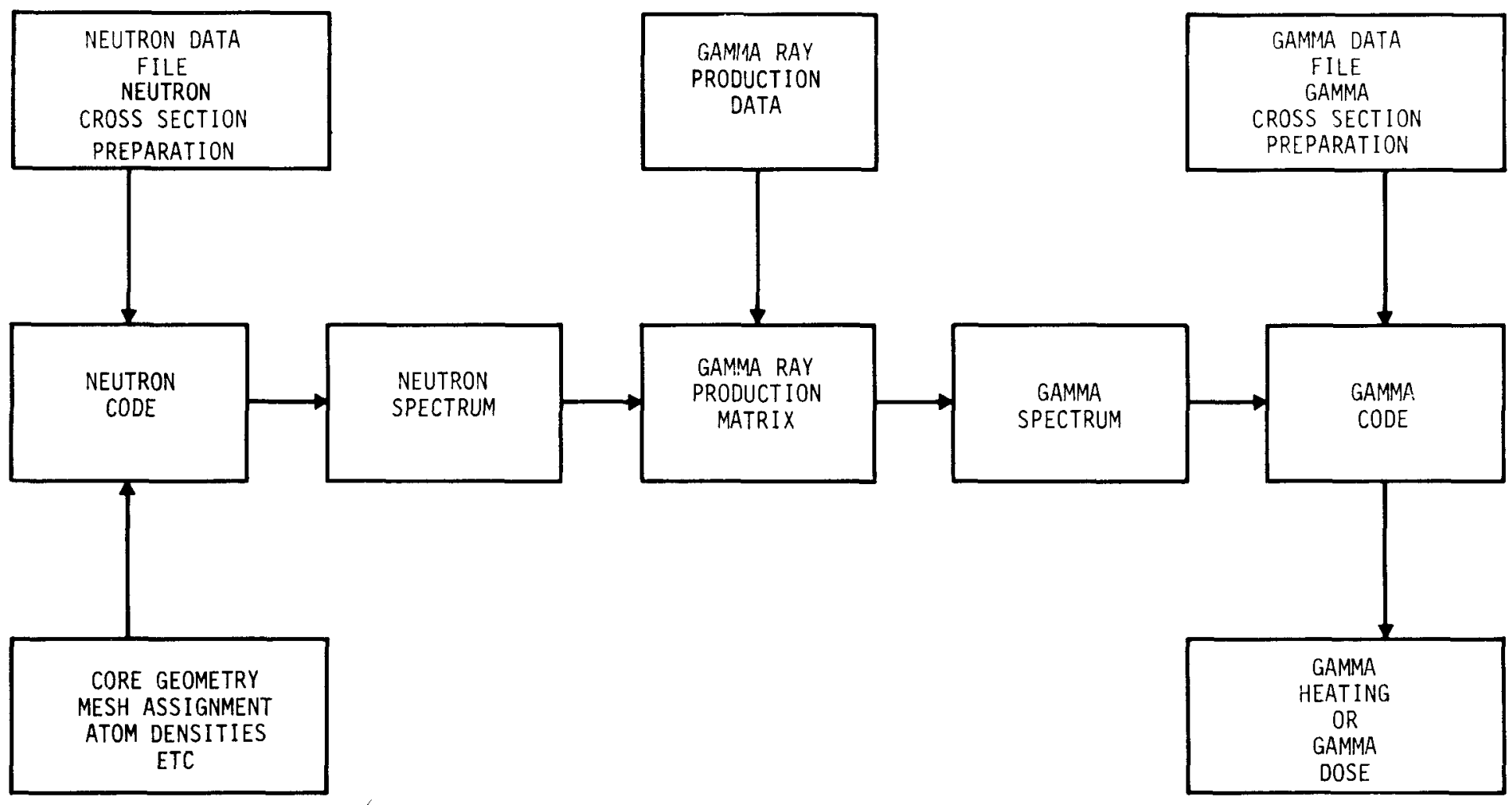

HEDL 7511-67.6 
proximity of facilities and intra-laboratory efforts. The computer codes used in gamma calculations at ANL and HEDL are compared in Table $V$.

In the main,* ANL analyses are based upon 2-D discrete ordinate transport theory using the computer code DOT as developed by Mynatt and co-workers at ORNL, whereas HEDL has employed 2-D and 3-D diffusion theory using computer codes 2DBS and 3DB. $(49,50)$ Cross section data are obtained from Evaluated Nuclear Data Files, Version B (ENDF/B) at various stages of evolutionary development (I through IV). The codes, $M c^{2(51)}$ and 1DX, (52) have been developed for preparing resonance shielded cross sections at ANL and HEDL, respectively. Basic gamma production cross sections in use at ANL are obtained from the POPOP4 code. (53) In contrast, HEDL uses essentially the survey work of Kidman (10) which, in turn, includes data from the POPOP4 library. The MUG code ${ }^{(54)}$ for preparation of gamma cross sections is used at both laboratories for subsequent transport or diffusion theory treatment of the generated gamma source spectrum. In order to assess these differences more clearly, a separate discussion of ANL and HEDL analytical work is warranted.

The current analyses performed at ANL stem from the early efforts of Miller to characterize ${ }^{(55)}$ and tailor ${ }^{(56)}$ the EBR-II gamma flux and heating environment. Transport theory (DOT) computations were conducted with 22 neutron groups and 20 gamma-ray groups in $X Y$ and/or RZ geometry. Subsequent work $^{(57)}$ expanded the neutron computations to 29 groups and used more recently compiled ENDF/B data as well as improved gamma-ray production cross sections. However, it is generally recognized that EBR-II neutronics codes are not completely adequate off mid-plane. (58) In fact, comparison of these calculations with observed reaction rates generally show increasing disagreement with increasing distance from mid-plane. (59) As a consequence, a primary input to the gamma calculation, namely the neutron spectrum becomes suspect for departures that are far from core mid-plane and in particular in blanket and reflector regions. Similar disagreements are also reported in the analyses

\footnotetext{
*Exceptions to this general conclusion do exist, i.e., 3-D diffusion theory calculations have been conducted by the EBR-II project and 1-D transport theory has been applied at HEDL. However, these exceptions represent very 1 imited efforts and therefore need not be considered here.
} 
TABLE V

GAMMA-RAY COMPUTER CODES

Computation Task

Neutron Data File

Neutron Cross Section

Preparation

Neutron and Gamma Codes

Gamma Ray Production Data

Gamma Ray Production Matrix

Gamma Data File

Gamma Cross Section Preparation
ANL

ENDF/B

$M c^{2}$

2D Transport Theory (DOT)

POPOP4

MUG
HEDL

ENDF / B

IDX

2D and 3D

Diffusion Theory

(2DBS and 3DB)

Kidman Survey ${ }^{a}$

MUG

${ }^{a}$ See Reference 10. 
of ZPPR control rod experiments, ${ }^{(60)}$ using essentially identical analytical procedures. Here again the comparison with TLD dose measurements for stainless steel is reasonably good $(210 \%)$ near core center but rapidly deteriorates with increasing distance from core center.

In contrast, the diffusion theory work at HEDL has utilized 42 neutron groups and 18 gamma-ray groups for 2-D ( $X Y$ and RZ) as well as 3-D ( $X Y Z$ ) calculations. Extensive comparison of the neutronic portion of these computations with reaction rate measurements in ZPR-9-EMC $(61)$ has shown generally good agreement within the core and poor agreement near the core extremities and at boundary regions. This type of behavior is to be expected of diffusion theory, which characteristically becomes a poor approximation in such regions. As a result, one must expect this deficiency to be directly reflected in any comparisons of calculated and measured gamma-ray phenomena. This is borne out in the analysis of TLD measurements in ZPR-9-EMC, (12) where in-core agreement rapidly deteriorates with increasing distance from core center.

A considerable component of this particular disagreement could well be absolute in nature, since experiments conducted in ZPR-3 mock-ups of EBR-II, viz. Assemblies 60 and 62 , reveal general agreement between calculations and TLD measurements for the relative radial distribution of gamma dose from core center out to the blanket region. $(53,63)$ For the steel reflected system (Assembly 62), the agreement in the shape of the relative radial gamma dose distribution is improved over the earlier work in the uranium reflected system (Assembly 60). The most recent TLD low power critical assembly measurements $(100)$ also exhibit good agreement with calculated radial distributions (see below). Hence, a significant aspect of any comparison between theoretical prediction and experimental observation of gamma heating must depend upon the absolute level (or fluence) of the in-core gamma-ray spectrum.

Comparisons between theory and experiment for reactor gamma-ray related phenomena must not only account for both absolute reactor power, but the previous power-time-history of reactor operation as well. In regard to absolute power normalization, three different methods have been used to compare calculations and measurements. Calculations at the EBR-II project have assumed a power level of $62.5 \mathrm{MW}$ which has subsequently been reduced by about $9 \%$ as a 
result of recent heat balance and flow rate measurements. Using this given absolute power, calculations are normalized so that the total gamma-ray energy deposition in the reactor is simply that energy fraction per fission expended in the form of gamma-rays. This procedure, first advanced by Miller, $(55,56)$ assumes negligible gamma leakage so that total gamma-ray energy deposition is therefore equal to total gamma-ray energy production. This normalization process is extremely advantageous in that the energy partitioning of the fission process is more accurately known than gamma spectra generated from computer codes which are not only subject to computational limitations, but must reflect inherent uncertainties in neutron and gamma-ray cross sections as well as in gamma-ray production modes.

In low power ZPR critical assembly comparisons, analytical work is carried out on a per fission basis, i.e. the neutron spectrum is normalized to yield a single fission event at a given point in the critical assembly (usually near core center). Absolute fission rate measurements (usually fission foil activations) are carried out at this point and thereby furnish the necessary normalization factor between theory and experiment.

For FFTF shielding studies in ZPR-9-EMC, the analytical work was normalized to the energy (power $x$ time) expended in the fast critical assembly irradiation. This procedure is subject to the largest uncertainty, since critical assembly power can not be measured with comparable accuracy to either a point fission rate or to absolute high power measurements, such as conducted in EBR-II.

The dependence of gamma calculations upon power-time reactor operating history has already been mentioned. Fission product decay gammas can comprise up to approximately $30 \%$ of the in-core LMFBR gamma intensity for sufficiently long reactor operation, i.e., when fission product build-up reaches equilibrium. In high power analyses such as for EBR-II operation, this time-history usually presents no problem since saturation of fission products is almost entirely complete within a few days, whereas run-to-run irradiations last roughly a month or so. However, this time-dependent contribution can be a particularly important factor in low power fast critical experiments, where irradiations normally last at most a few hours and equilibrium conditions are not attained. 
Since gamma-ray calculations customarily invoke equilibrium conditions, it may be imperative to correct experimental data to account for this effect prior to any comparison with calculation.

In 211 these computer codes, ${ }^{235} \mathrm{U}$ fission product decay gammas are used discounting any differences which exist amongst those fissile nuclides ${ }^{238} \mathrm{U}$, ${ }^{239} \mathrm{Pu},{ }^{240} \mathrm{Pu},{ }^{241} \mathrm{Pu}$... etc., which arise in LMFBR. In absence of actual observations for these nuclides, this assumption is entirely reasonable. It does, however, serve as a particularly good illustration of the need for an expanded nuclear data base for gamma calculations. While all reactor calculations suffer from this limitation, gamma-ray computations possess even greater shortcomings in this regard. Gamma calculations must apriori possess all such existing inadequacies of neutron calculations and in addition suffer from important deficiencies in basic data for the following gamma-ray production modes:

1) Fission product decay

2) Prompt fission

3) Neutron capture

4) Inelastic neutron scattering

In addition to the deficiency in fission product decay gamma radiation, a parallel data gap has existed in the prompt fission gamma spectra of the fissile nuclides that arise in LMFBR. Hence all calculations to date have utilized the prompt fission gamma spectrum of ${ }^{235} \mathrm{U}$. Measurements of prompt fission gamma spectra for ${ }^{239} \mathrm{Pu}$ and ${ }^{240} \mathrm{Pu}$ have just become available and have been added to the gamma production libraries of some codes. However, these data have not actually been used as yet and therefore do not bear upon any comparisons cited in this review.

It is well known that the spectra of gamma-rays emitted in neutron capture is a very sensitive function of neutron energy. Branching ratios change rapidly with nuclear excitation energy and different levels in the compound nucleus can be reached by fast neutrons as opposed to thermal neutrons. Nonetheless, the data libraries of all of these gamma codes rely essentially on 
thermal neutron induced capture gamma spectra, owing to the virtual nonexistence of energy dependent data.

Recognizing these serious deficiencies in the basic nuclear data for gamma calculations, Kidman (10) advanced a normalization technique which conserves total gamma-ray energy released in both fission and capture modes. For a capture process, instead of the exoergic $Q$ of a given reaction, one should use $Q+E_{i}$, where $E_{i}$ is the neutron energy of the $i^{\text {th }}$ group.* For fission, one increases the total (thermal) gamma energy yield by the factor $\left[1+\left(E_{j} / T\right)\right]$, where $T$ is the total energy released in thermal fission. It is interesting to note that Kidman's normalization technique can be viewed as a microscopic analogue of Miller's normalization method, since the latter is carried out at a macroscopic level for the entire reactor. It is clear that both these methods were independently motivated to improve gamma calculations by attempting to circumvent current inadequacies of required nuclear data.

Various improvements have been described for both experimental and theoretical techniques, which render all but the most recent work obsolete. Hence only the latest comparisons between theory and experiment will be considered. At high power, the most recent analysis of EBR-II gamma heat calorimetry in S/A XX05 ${ }^{(13)}$ yields an average calculated-to-experimental (C/E) ratio of $1.04 \pm 0.02$ for observations carried out in 16 separate EBR-II runs (\#60-\#74). This is reasonably good agreement, considering calorimetry measurements possess about 7\% error (see Section II.A. 1) and calculational errors are probably larger (see above). In fact, provided that macroscopic energy normalization is applied, these calculational errors have been assessed at about $10 \%$ with the major uncertainties attributed to the neutron flux, gamma production and transport cross sections, and local depression of gamma flux. It must be stressed that the above comparisons apply only to gamma heating in stainless steel. In this regard an additional systematic effect is currently under study at the EBR-II project which would alter the above cited C/E ratio of 1.04 , namely direct neutron heating through elastic scattering. (64) It has

*Actually the increase in energy available for gamma production is not exactly $E_{j}$, but rather is the center-of-mass energy $E_{j} /\left(1+A^{-1}\right)$, where $A$ is the mass number of the interacting (target) nucleus. 
a 7 ready been mentioned that this effect can contribute as much as $12 \%$ to ${ }^{7}$ LiF TLD response. Direct neutron heating has also been estimated in analytical studies pursued to evaluate europia as an LMFBR control rod alternative. In these studies, $(10,11)$ the direct neutron heating in europia has been calculated to range from 3 to $5 \%$. In view of the mass number $(A)$ dependence of direct neutron heating, it is not unreasonable to expect roughly the same order of magnitude for direct neutron heating in stainless steel. Since direct neutron heating is an inherent contributor to calorimetry measurements, the corrected C/E ratio (involving gamma heating only) would necessarily be higher and can conservatively be set at approximately 1.1 .

The most recent TLD measurements in $2 P P R(40)$ provide radial gamma heating distributions in stainless steel for Assembly 4 - Phase 1. About 80 stainless steel encapsulated 7 LiF TLD specimens were used at locations extending from core center out to the blanket region. Excellent agreement with calculations was obtained, especially considering the variation of gamma spectra encountered at all these locations. The average $C / E$ ratio for all these measurements is quite close to unity $(\sim 1.01 \pm 0.03)$ and represents essentially ideal agreement in view of the statistical precision of the TLD technique ( $23 \%)$.

It is difficult to explain this remarkable result. Recall that TLD measurements possess an absolute accuracy of $210 \%$, under the most favorable conditions. (See Section II.B.1.) While dosimeters consisting of ${ }^{7} \operatorname{LiF}(\bar{Z} \approx 8.2)$ surrounded by stainless steel $(\bar{Z} \approx 26)$ do not represent a well matched system, the spectrally averaged correction factors $f^{-1}$ are close to unity for the entire domain of gamma spectra encountered. In fact at all these TLD locations, this correction factor 1 ies in the range $0.95 \leq f^{-1} \leq 1.05$, so that favorable conditions do exist for such TLD measurements.

On the other hand, the accuracy of in-core gamma heating calculations for LMFBR have been assessed between $10 \%$ to $25 \%$ by various workers and groups since the initiation of these analyses some 5 years ago. Unfortunately the ZPR-ANL group have not assigned or reported any specific accuracy for their calculations. Since their work parallels that of the EBR-II project quite closely, one can assume the most recently quoted accuracy for EBR-II calculations without macroscopic energy normalization is also applicable here, namely 
$25 \%$. Faced with approximate uncertainties of $25 \%$ and $10 \%$ in theory and experiment, respectively, one wonders whether the remarkable agreement attained here is generally representative of LMFBR environments. Only time will tell, provided sufficient programmatic support is furnished to stimulate significant improvement in both experimental and calculational techniques.

In experimental studies of LMFBR control rod materials ( $B_{4} C$ and $\left.E U\right)$, ${ }^{7}$ LiF TLD measurements were carried out in ZPR-9-EMC. Analyses of these critical experiments were recently performed at HEDL $(11)$ using gamma-ray calculations based on diffusion theory (as outlined above). This comparison yielded a $C / E$ ratio of approximately 1.1 for gamma-ray heating in europia. Based on this agreement together with improved Eu neutron cross sections, the accuracy of HEDL gamma calculations was inferred to be less than the originally assigned estimate of $\sim 25 \%(10)$ and was assessed to $1 \mathrm{ie}$ in the range between $10 \%$ to $20 \%$. It is felt that such a recommendation is overly optimistic because:

1) This C/E ratio is based upon only a very limited number of points in just one critical assembly experiment.

2) The analytical treatment neglects gamma-ray production from inelastic neutron scattering, which can comprise roughly $15 \%$ to $20 \%$ of the total gamma-ray energy released in LMFBR.

3) Additional uncertainties exist in low power critical experiments due to absolute power normalization and possible nonequilibrium exposure to fission product decay gammas. The latter correction is substantially reduced in critical assembly experiments by waiting a day or more after shutdown before removal of the TLDs. In this manner the TLD exposure is within a few percent of a comparable irradiation under equilibrium conditions. Unfortunately, the accuracy of absolute power attained in fast critical assembly experiments is not known nearly as well. It is customarily determined by volume integration based on absolute fission rate measurements made at a limited number of space points together with relative fission rate traverses throughout the critical assembly. The uncertainty in this procedure for ZPR type critical experiments has not been reported, but probably lies somewhere in the $5 \%$ to $10 \%$ range. 
Unfortunately all of these effects imply an increase in overall uncertainty. Moreover, the largest effect, i.e., (2) is systematic and when taken into account produces a re-estimated C/E ratio of roughiy 1.25 . Consequently, the initial uncertainty estimate of $225 \%$ originally cited for HEDL computations would still appear to be valid.

In summary, the three most recent comparisons between theory and experiment in LMFBR environments at EBR-II, ZPPR, and ZPR-9-EMC give corrected C/E ratios of about $1.1,1.0$, and 1.25 , respectively. In view of the large variation in uncertainty currentiy assigned to calculations alone, 10-25\%, these results are certainly plausible. 


\section{RECOMMENDATIONS}

General observations on the "state-of-the-art" will be considered prior to presenting any specific recommendations. These overall conclusions are important in their own right and form the proper perspective for specific recommendations, which are advanced separately for experiment and theory below.

Within the last five years, progress has been made in defining the effects of gamma radiation in LMFBR environments. Nevertheless, it is clear that the current "state-of-the-art" falls considerably short of current and requested FFTF goal requirements. These demanding goals cannot be attained without proper support for dedicated efforts in both theory and experiment. The responsibility for this work must be clearly delineated and fully supported. Progress exhibited by the EBR-II project provides a realistic basis to forecast future FFTF needs. Competent analytical and experimental staffs will be required to rigorously define irradiation environments in order to design, tailor, conduct, and evaluate experiments at projected goal accuracies. The details of high power LMFBR environmental characterizations, as confronted in FFTF irradiations, can not be left in the hands of metallurgy or materials specialists. These workers cannot be expected to have a competency so broad as to fulfill the total needs of any given in-core materials irradiation test. Moreover, they are sufficiently occupied in their own specializations, otherwise they could not plan and conduct these costly radiation damage experiments with the required materials expertise.

Expert reactor analysts and experimentalists must provide the detailed environmental data and predictions required of future FFTF operations and experiments. Not only will current methods be required, but development and innovation of techniques will be demanded by the complexities of the FFTF reactor operations and the specific needs of given experiments. This completely parallels the demands placed upon the analytical staff of the EBR-II project. A comparable demand will also exist for the development, improvement, and application of experimental techniques to measure gamma heating and temperature in FFTF, be they active or passive methods. In direct analogy to analytical efforts, much more is required beyond merely the rote application 
of already existing experimental methods. Competent personnel must be assigned on a long term basis. Otherwise solutions for day-to-day FFTF problems, whether they be experiment design, reactor operations, or postirradiation analyses, may go wanting and the overall quality of the entire program could easily be compromised. The very raison d'être for FFTF clearly demands that appropriate and responsible action be promptly instituted.

A. Development of Experimental Techniques

A major necessity of high power LMFBR experiments is the time-history of the temperature, $T(t)$, during the irradiation. Current passive monitors cannot supply such time-dependent data; nor have any concepts been advanced which possess this capability. The discovery and validation of such a technique at this time would necessarily be classified as a major breakthrough in research reactor technology. A long term base of support should be provided so that competent reactor experimentalists can be stimulated to attack this problem area with the vigor it rightfully deserves.

1. Calorimetry

Three specific recommendations have already been given in Section II.A.l for improving gamma heat calorimetry, viz. pg. 9a, b, and c. It should be possible to incorporate all three modifications in the next generation of calorimeters and thereby reduce experimental error from the current level of about $7 \%$ down to the $3-5 \%$ range. Additional incentive exists for the third recommendation, namely calorimeter construction from additional materials and elements. The systematic Z-dependence of gamma heating would be of general value in planning and designing in-core experiments. Besides furnishing incore heating measurements in materials of interest other than stainless steel, this extension would establish a broader base for testing calculations, since gamma heat deposition is a strong function of atomic number $(Z)$. This concept is somewhat analogous to the use of different reaction rates (neutron cross sections) to provide integral measurements that can be used to investigate predicted neutron spectra. For gamma-rays, different integral measurements can be obtained from calorimetry with materials of sufficiently different $Z$. These data could then be used to investigate the validity of calculated incore gamma spectra. 


\section{Ionization Chambers}

Although ionization chambers have not been effectively applied in either high or low power US-LMFBR environments, they afford an important active method which has been used to compliment calorimetry. Ionization chambers have been constructed from many different wall materials and filling gases. However, for sma11-cavity ionization theory to be valid, one must again have a well-matched system, i.e., the atomic number $(Z)$ of the chamber wall material should be approximately the same as that of the filling gas. A particularly well-matched system is a graphite walled ionization chamber filled with $\mathrm{CO}_{2} \cdot(9,20)$ Chambers have been fabricated that are small enough for in-core use and operate acceptably up to roughly $900^{\circ} \mathrm{F}$. (65) Such ionization chambers would possess limited ability to cover the complete temperature range encountered in EBR-II or FFTF experiments. Consequently, some development work to extend this upper temperature limit is warranted. The capability of these ionization chambers to withstand high fluence fast neutron irradiation and still maintain an acceptable performance level is perhaps a more fundamental limitation that should be investigated. Just as in calorimetry, fast neutron induced ionization chamber response would depend primarily upon atomic number $(A)$ and should be taken into account.

\section{TED Monitors}

Of all the passive monitors, TEDs currently yield the most accurate and reliable temperature measurements. They suffer, however, from the important limitation of radiation induced swelling and as a consequence can be in error for high fluence experiments above roughly $10^{22} \mathrm{n} / \mathrm{cm}^{2}$. It has already been recommended in Section II.A.2 that the TED technique be further developed by using swelling-resistant materials such as Inconel 600 or 625 to replace the stainless steel 304 in present use. In addition, the possibility of ascertaining correction factors to account for this effect should be actively pursued. The recent example of "dummy" TEDs filled with helium instead of sodium is only one of perhaps a number of different approaches to determine accurate correction factors. 


\section{VAP Monitors}

Negligible fluence sensitivity is the major advantage claimed for the VAP passive temperature monitor. Since many future experiments will be conducted at very high fluence in both EBR-II and FFTF, the unique potential of this method should not be overlooked. Little, if any, work has been done on VAPs since this concept was originally advanced. Radiation damage of the quartz chamber wall for fast fluences greater than $5 \times 10^{21} \mathrm{n} / \mathrm{cm}^{2}$ could be a significant limitation that should be investigated. This method as well as any promising alternatives for passive temperature monitoring at high fluence should be systematically investigated and tested.

\section{TLD Dosimeters}

A number of recommendations have already been advanced in Section II.B. 1 for improving TLD gamma-ray dosimetry. Fast neutron sensitivity of currently manufactured and used ${ }^{7}$ LiF rods must be accurately measured. The TLD technique should be compared with other gamma-ray dosimetry methods and in particular with absolute gamma-ray dosimetry by recoil electron spectroscopy. (See Section II.B.2.) Such a comparison would permit systematic study of the correction factor $\left\langle f^{-1}\right\rangle$ for a wide variety of materials in LMFBR environments and also furnish an independent means of absolute calibration for TLD dosimeters. Significant improvement in overall accuracy should no doubt result. While a quantitative forecast is inopportune at this time, reduction of overall uncertainty from the current $210 \%$ level down to roughly $5 \%$ would seem plausible.

\section{Compton Recoil Gamma-Ray Spectroscopy}

The absolute gamma-ray energy spectrum is the most fundamental quantity in the specification of a gamma radiation field and any induced effects arising therefrom. Accurate prediction of this spectrum is the goal of calculational methods. Yet, surprisingly, gamma-ray spectral comparisons between theory and experiment for LMFBR environments are nonexistent. Only one method has evolved to date with the ability to measure low power in-core gamma continua, namely Compton Recoil Gamma-Ray Spectroscopy. This unique method has unfortunately not been applied in spite of the obvious benefits 
that would accrue to both calculations and other (integral) gamma-ray dosimetry methods. Compton Recoil Gamma-Ray Spectroscopy has not been actively pursued and therefore has not attained near the potential applicability it possesses for in-core reactor measurements. A development program to fully exploit this potential is clearly warranted. In particular, extension of the upper energy limit of applicability would be highly desirable. It is high time that this method be applied in low power LMFBR mockup experiments. Such measurements will furnish sorely needed data for comparison with calculations at the most fundamental level.

B. Development of Calculational Methods

1. Basic Nuclear Data

A11 reactor physics analyses suffer from uncertainties that exist in the fundamental data, such as cross sections, energy spectra, yields, halflives ... etc. Gamma calculations are obviously no exception. In that the source spectrum of gamma rays depends intimately upon the space and energy distribution of neutrons, gamma calculations must inherently possess those limitations arising in the neutronic computations that are employed. Beyond those shortcomings in common with neutron calculations, there exist uncertainties in gamma-ray production and transport cross sections as well as additional quantitative deficiencies in gamma emission and energy release in the fission process. ${ }^{235} U$ data is almost universally used for all fissile nuclides of importance in LMFBR. Due to lack of data on other fissile isotopes, the energy spectrum of both prompt and delayed (fission product) gammas as well as the time dependence of delayed gammas is assumed to be that of ${ }^{235} \mathrm{U}$ regardless of the core loading. Prompt and delayed fission gammas account for at least $60 \%$ of the total gamma-ray energy release in LMFBR. Deficiencies of this kind for ${ }^{239} \mathrm{Pu}$ are unacceptable and must be amended if the current accuracy of gamma calculations is to show any improvement.

In neutron capture, the rapid dependence of gamma-ray emission spectra on neutron energy has already been pointed out along with serious uncertainties introduced by using thermal capture gamma-ray spectra for fast reactor computations (see Section III). Actually all gamma production modes are to 
some extent neutron energy dependent, with the capture process exhibiting the greatest energy dependent variability. An ideal data bank would comprise the mono-energetic dependence of neutron capture gamma-ray spectra for all the elements, so that the analyst could properly account for this energy dependence. As such a prospect is completely out of the question, one has no other recourse except to resort to more pragmatic experimental solutions that will supply, in principle, improved basic data for fast reactor gamma calculations. Such an experimental program is outlined in Section $C$ below.

\section{Analytical Techniques}

Reliance upon diffusion theory as opposed to transport theory for both gamma and neutron calculations is perhaps the outstanding feature of FFTF analyses at HEDL. In contrast, the EBR-II project at ANL has depended, in the main, on transport theory. This difference is entirely reasonable, since FFTF is much larger than EBR-II and extensive application of transport theory is therefore unwarranted in most instances. Moreover, the 3-D modelling capability currently available with diffusion theory codes is not available or even economically feasible with transport theory. Diffusion theory does, however, possess limitations, in particular at boundary regions and near core extremeties. These restrictions can arid should be quantitatively defined by appropriate comparisons with both transport theory and experiment. Unfortunately, experimental data are hard to come by for high power environments, such as found in EBR-II and FFTF. Hence, "state-of-the-art" capability with existing transport theory codes will be required of the FFTF analytical staff in order to validate the applicability and limitations of 3-D diffusion theory. As stressed earlier, these efforts are far more than mere rote application of existing codes, as attested to by experience already gained from EBR-II operations.

Calculations to date, whether diffusion or transport theory, have used no more than 20 groups or so in the treatment of the gamma spectrum. It would be instructive to ascertain the dependence of predicted gamma heating (for high power calorimetry experiments) and gamma dose (for low power critical assembly experiments) upon gamma group structure. As a first step, comparison with calculations using roughly 30 groups would appear feasible. 
Additional incentive to increase the number of gamma groups in calculations will no doubt be derived through direct comparisons with measured spectra. Here the capability of Compton Recoil Gamma-Ray Spectroscopy for observing fine structure in the gamma continuum has already been emphasized (see Section II.B.2). In fact, the actual choice of gamma group structure in calculations should be based on features revealed in these higher resolution measurements. It would be productive in this regard to carry out calculations for the single already existing measurement of a fast reactor gamma spectrum, namely for ZPR-6, Assembly No. 6.

All gamma calculations should simultaneously invoke the macroscopic and microscopic energy normalizations first introduced by Miller and Kidman, respectively. Improvements in the systematics of energy partitioning for fission and capture processes should be incorporated in code libraries as they become available for nuclides of interest in LMFBR environments. In absence of these normalizations, theoretical results depend on errors in more poorly known gamma production and transport cross sections, which in turn are subsequently compounded by propagation through computations. Hence it is anticipated that overall uncertainty introduced by these energy normalizations will continue to be significantly smaller.

It has already been remarked that direct neutron heating through elastic scattering is not completely negligible for many materials of interest. This effect has been included in the calculations of some but not all workers. Since this source of heat generation can be readily computed from the calculated neutron spectrum, it is recommended that this effect be evaluated in all gamma calculations.

C. Specific Experiments

1. In-Core Gamma-Ray Spectrometry

The necessity for gamma-ray spectral measurements in low power LMFBR mockups such as ZEBRA, ZPR, ZPPR ... etc., as well as in standard radiation fields such as CFRMF, $\Sigma \Sigma \ldots$ etc., is two fold. From a more fundamental viewpoint, our quantitative knowledge of LMFBR environments has and will continue to be seriously deficient in absence of in-core gamma spectral 
measurements. The inter-relationship and complementarity of in-core reactor radiation field components has already been discussed. On the other hand, lacking these fundamental measurements, progress will continue to languish in both calculational methods and integral dosimetry measurements, (e.g., TLD). From a more pragmatic viewpoint, it is the evolution of accurate calculational tools developed in low power experiments and analyses which bear directly upon the effective use of EBR-II and FFTF.

The unique ability of Compton Recoil Gamma Ray Spectroscopy to fill this void has already been emphasized. As a general rule, in-core gamma spectra should be routinely measured in all LMFBR related low power mockups, just as now become the custom for neutron spectrum measurements. Specific gamma spectra can and should be measured in special fast critical facilities designed to test the adequacy of both transport and diffusion theory. Such experiments should cover a broad domain of variation in gamma spectra and extend from the core center out to blanket and reflector regions. TLD measurements should be carried out at the same locations so that intercomparisons between integral measurements, differential measurements, and calculations are simultaneously possible. Spectral measurements in special out-of-core experiments and calibrations should be performed to reduce overall absolute error of the TLD technique.

\section{Gamma-Ray Production Measurements}

The virtual nonexistence of energy dependent gamma-ray production cross sections creates a significant gap in the basic data required for fast reactor gamma calculations. It has already been surmised that detailed neutron energy dependence for important gamma-ray production modes is at best a dream devoutly to be wished. In reality, many, many years may pass before basic data of such detailed nature becomes available. As a practical alternative to the current use of thermal data, an experimental program should be launched to generate fast neutron spectrum averaged gamma-ray production modes for gamma calculations. To this end, a broad range of LMFBR type environments, covering a significant variation in fast neutron spectra, should be employed. 
Fast critical facilities, both here and abroad which have been adapted for pulsed neutron time-of-flight (TOF) spectrometry, are well suited for this task. The existing TOF re-entrant channel can be used for collimation in pulsed or steady-state leakage gamma measurements. For these experiments, it would be desirable to void the entire in-core channel extending through the critical assembly. Gamma leakage spectra can be measured with and without samples of interest placed at the core center in the voided channel.* $A$ completely voided channel would reduce dependence on gamma background spectra. Both the pulsed and steady-state critical assembly operation would be advantageous, depending on the type of sample introduced. For non-fissile samples, gamma production modes (e.g., capture and inelastic scattering) are virtually instantaneous so that pulsed measurements would reduce even further corrections entailed from uncorrelated gammas. For fissile samples, measurements could be made at steady-state so that equilibrium conditions are established for fission product decay gammas. In these latter experiments, all other production modes, e.g., prompt fission gammas, capture gammas ... etc., would also be contributing to observed leakage spectra.

A series of experiments of this type should be conducted covering all LMFBR environments of interest. Such a program would allow effective energy dependence of gamma-ray production modes to be assessed. More realistic basic data would be generated for direct use in calculations. As a natural consequence, significant improvement in the accuracy of fast reactor gammaray calculations would automatically accrue.

\footnotetext{
*Any survey of potential detection schemes for these experiments lies well outside the scope of this review.
} 


\section{Summary}

Many detailed recommendations have been advanced to improve the "stateof-the-art" in gamma-heating characterization of LMFBR. Considerable effort in these diverse areas will be required in order to attain LMFBR program objectives. Some estimate of the overall effort involved can be gained from inspection of Table VI, which summarizes the present status of selected aspects of this work in contrast with current and requested goal accuracies. In this context, current goal accuracies are considered realistic, i.e. are thought to be attainable in the near future. Requested goal accuracies are long range and more in the nature of future ideal goals.

Current goals are generally a factor of two or so better than present accuracies, whereas requested goals are roughly a factor of three better than the presence "state-of-the-art". It is possible to achieve most if not all of these current goals provided a well planned program is launched with identified responsibilities and properly sustained support. Initiation of such a program in the immediate future would provide substantial benefits at a very opportune time, i.e. prior to the first full scale experiments in FFTF. 
TABLE VI

ACCURACY OF SELECTED LMFBR GAMMA-HEATING ACTIVITIES

Activity

High Power

Calorimetry

Passive Temperature Monitor (TED)

Low Power

Dosimetry (TLD)

Low Power

Spectrometry

Calculations
Present Accuracy

$7 \%$

$10-20^{\circ} \mathrm{F}$

$10 \%$

$20 \%$

$10-25 \%$

\section{Goal Accuracy}

Current Requested

$5 \%$ $<3 \%$

$<10^{\circ} \mathrm{F}^{\mathrm{a}}$ $5^{\circ} \mathrm{F}^{\mathrm{a}}$

$5 \%$

$<3 \%$

$5 \%$

$<3 \%$

$5 \%$ $<3 \%$

${ }^{a}$ Desired accuracy for high fluence irradiations. 


\section{REFERENCES}

1. H. Greenspan, C. N. Kelber, D. Okrent (Eds.), Computing Methods in Reactor Physics, Gordon and Breach Publishers, New York, N.Y. (1968).

2. W. N. McElroy, "Fast Reactor Flux - Spectral Characterization," Proc. Am. Nuc. Soc. Topical Meeting on Irradiation Experimentation in Fast Reactors, Jackson, Wyoming (1973).

3. B. R. Sehgal and D. Meneghetti, "Analys is of Dosimetry Measurements in EBR-II and EBR-II ZPR-3 Critical," Proc. Am. Nuc. Soc. Topical Meeting on Irradiation Experimentation in Fast Reactors, Jackson, Wyoming (1973).

4. W. N. McElroy, R. A. Bennett, D. L. Johnson, and N. D. Dudey, "Neutron Environmental Characterization Requirements for Reactor Fuels and Materials Development and Surveillance Programs," Ist ASTM-EURATOM Sym. on Reactor Dosimetry, Petten (1975).

5. R. Gold, "Neutron Spectrometry for Reactor Applications: Status, Limitations and Future Directions," Ist ASTM-EURATOM Sym. on Reactor Dosimetry, Petten (1975).

6. C. H. Hogg, "Gamma Heating Measurements in the MTR," IDO-16093 (1953).

7. W. F. Witzig, "WAPD-1 Experiments in the MTR," WAPD-79 (1953).

8. C. H. Hogg, "Gamma-Ray Dosage Measurement," ID0-16205 (1954).

9. L. D. Weber and C. H. Hogg, "MTR Gamma Heat Generation Measurements," IDO-16652 (1961).

10. R. B. Kidman, "FTR Europa Heating Rates," HEDL-TME 74-20 (1974).

11. J. T. Ward, "Gamma Heating in FTR Europa Control Assemblies," HEDL-TME 75-35 (1975).

12. E. T. Boulette, D. R. Marr, and W. L. Bunch, "Analysis of the Measured Gamma Dose Rate Distribution in the FTR/EMC," HEDL-TME 73-42 (1973).

13. R. A. Laskiewicz, J. F. Koenig, and E. R. Gilbert, "Measurement and Calculation of Gamma Heat in an Instrumented Subassembly in EBR-II," Trans. Am. Nucl. Soc. 22, 685 (1975).

14. A. E. Arave and R. H. Meservey, "A High Temperature U1trasonic Thermometer for Measuring Reactor Fuel Temperature," IN-1413 (1970).

15. A. E. Arave, "A High Temperature U1trasonic Thermometer for In-Reactor Fuel Rod Centerl ine Temperature Measurements," ANCR-1129 (1973).

16. R. A. Laskiewicz and J. F. Koenig, "Comparisons of Measured with Calculated Gamma Heating in Subassembly XX05," ANL-RDP-39 (1975). 
17. Y. S. Touloukian (Ed.), Thermophysical Properties of Matter, Vo1. 7, Plenum Press, New York (1970).

18. Y. S. Touloukian (Ed.), Thermophysical Properties of Matter, Vol. 1, Plenum Press, New York (1970).

19. A. W. Boyd, (Ed.), Determination of Absorbed Dose in Reactors, IAEA Tech. Rep. -128 , Vienna (1971).

20. A. W. Boyd, "Dosimetry Methods for Structural Materials: Nuclear Heating," ist ASTM-EURATOM Sym. on Reactor Dosimetry, Petten (1975).

21. E. R. Specht, T. H. Springer, and J. M. Otter, "Heating Rates and Analysis for Alternate Control Assemblies," AI-ERDA-13153 (1975).

22. J. L. Straalsund, R. L. Fish, P. K. McDaniel and W. V. Cummings, "Irradiation Temperature in Row II of EBR-II," HEDL-TME 71-91 (1971).

23. G. L. Hofman, "An In-Reactor Temperature Monitor," Nucl. Technol. 19, 204 (1973).

24. D. G. Frankl in and W. E. Ruther, "A Reactor In-Core Temperature Monitor," Trans. Am. Nucl. Soc. 14, 632 (1971).

25. C. C. Price, D. G. Franklin, and A. Gopalakrishnan, "In-Core Monitoring in the Experimental Breeder Reactor-II," Proc. Am. Nucl. Soc. Topical Meeting on Irradiation Experimentation in Fast Reactors, Jackson, Wyoming (1973).

26. W. R. Wallen, "Test of Temperatuare Monitors for Gamma-Heating Experiments," ANL-RDP-2 (1972).

27. J. F. Bates and T. K. Bierlein, "Characterization of the In-Reactor Environment of Subassemblies X-216 and X-223," HEDL-TME 75-77 (1975).

28. J. L. Gillette, "Preliminary Examination of the Thermal-ExpansionDifference Temperature Monitors in Subassembly XX07," ANL-RDP-41 (1975).

29. W. E. Ruther, "New Materials for TED Passive Temperature Monitors," ANL-RDP-33 (1974).

30. C. C. Price, "In-Core Gamma Calorimeter," ANL-RDP-41 (1975).

31. J. R. Cameron, N. Suntharalingam, and G. N. Kenney, Thermoluminescent Dosimetry, Univ. of Wiscons in Press, Madison (1968).

Z. Spurry and J. Sulcova, "Bibliography of Thermoluminescent Dosimetry," Health Phys. 24, 573 (1973). 
32. G. S. Stanford and T. W. Johnson, "Determination of Gamma-Ray Heating in a Critical Facility by Thermoluminescent Dosimetry," ANL-7373 (1968).

33. J. Adamson, R. M. Absalom, A. B. Baker, G. Ingram, S.K.I. Pattenden, and J. M. Stevenson, "ZEBRA6: Dilute Plutonium Fueled Assembly," Proc. Int. Conf. on Fast Critical Experiments and Their Analysis, ANL-7320 (1966).

34. E. T. Boulette and W. L. Bunch, "Analysis of ZPPR/FTR Shield Experiments: Gamma Distribution," WHAN-FR-13 (1971).

35. G. C. Simons, D. G. Stenstrom, D. Meneghetti and W. P. Keeney, "GammaRay Dose Evaluations for the ZPR-3/EBR-II Critical Assemblies", Trans. Am. Nucl. Soc. 13, 880 (1970).

36. S. G. Carpenter, G. G. Simons, A. P. 01son, R. W. Goin, and J. P. Plummer, "Boron and Tantalum Control Rod Studies and Absolute Gamma-Ray Absorbed Doses in a LMFBR Critical," Proc. Am. Nucl. Soc. Topical Meeting on New Developments in Reactor Physics and Shielding, Kiamesha Lake, New York (1972).

37. G. G. Simons and T. J. Yule, "Gamma-Ray Heating Measurements in ZeroPower Fast Reactors with Thermoluminescent Dosimeters," Nucl. Sci. Engng. 53, 162 (1974).

38. C. L. Wingate, E. Tochilin, and N. Goldstein, "Response of LiF to Neutrons and Charged Particles," USNRDL-TR-909 (1965).

39. T. E. Burlin, "Cavity-Chamber Theory", in Radiation Dosimetry, Vol. I, Chap. 8, F. H. Attix and W. C. Roesch, Eds., Academic Press, New York (1968).

40. G. G. Simons and C. L. Beck, "Radial Gamma-Ray Heating in ZPPR Assembly 4 Phase-1," ANL-RDP-35 (1974).

41. M. G. Silk, "The Energy Spectrum of the Gamma Radiation in the Daphne Core," J. Nucl. Energy 23, 308 (1969).

42. R. Gold, "Compton Recoil Gamma-Ray Spectroscopy," Nucl. Instr. and Meth. 84, 173 (1970).

43. R. Gold, "Gamma-Continuum at the Air-Land Interface," Health Phys. 21, 79 (1971).

44. R. Gold, A. M. Strash, F. J. Congel, and J. H. Roberts, "Continuous Gamma-Ray Spectroscopy in the Natural Environment," IEEE Trans, $\underline{\text { NS-20 }}$ 48 (1973).

45. R. Gold, B. G. 01 tman, K. F. Eckerman, and A. M. Strash, "Environmental Radiation at the EBR-II Site", IEEE Trans. NS-21, 596 (1974).

46. R. Gold, "Compton Recoil Measurement of Continuous Gamma-Ray Spectra," Trans. Am. Nuc1. Soc. 13, 421 (1970). 
47. R. Gold and A. M. Strash, "Gamma-Ray Dosimetry for the Natural Environment," Trans. Am. Nucl. Soc. 14, 502 (1971).

48. A. M. Strash and R. Gold, "Absolute Gamma-Ray Dosimetry by Recoil Electron Spectroscopy," Nature 234, 260 (1971).

49. W. W. Little, Jr., and R. W. Hardie, "2DB, A Two-Dimensional Diffusion Burnup Code for Fast Reactor Analysis," BNWL-640 (1968).

50. R. W. Hardie and W. W. Little, Jr., "3DB, A Three-Dimensional Diffusion Theory Burnup Code," BNWL-1264 (1970).

51. B. J. Toppel, A. L. Rago and D. M. O'Shea, "Mc ${ }^{2}$, A Code to Calculate Multigroup Cross Sections," ANL-7318 (1967).

52. R. W. Hardie and W. W. Little, Jr., "1DX, A One-Dimensional Diffusion Code for Generating Effective Nuclear Cross Sections," BNWL-954 (1969).

53. W. E. Ford, III and D. H. Wallace, "POPOP4, A Code for Converting GammaRay Spectra to Secondary Gamma-Ray Production Cross Sections," CTC-12, ORNL (1969).

54. J. R. Knight and F. R. Mynatt, "MUG, A Program for Generating Multigroup Photon Cross Sections," CTC-17, ORNL (1970).

55. L. B. Miller, G. H. Golden, R. E. Jarka, and K. E. Phillips, "Characterization of the Power in an Experimental Irradiation Subassembly of MixedOxide Fuel in EBR-II," ANL/EBR-047 (1971).

56. L. B. Miller and R. E. Jarka, "Local Modification of Irradiation Conditions," ANL/EBR-035 (1971).

57. D. G. Stenstrom, "Calculations of Gamma Heating in a Fast Reactor Environment" Trans. Am. Nuc1. Soc. 15, 551 (1972).

58. D. Meneghetti and W. B. Loewenstein, "EBR-II Irradiation Physics," Proc. Am. Nucl. Soc. Topical Meeting on New Developments in Reactor Physics and Shielding, Kiamesha Lake, New York (1972).

59. 3. R. Sehgal and F. H. Rempert, "Analyses of Dosimetry Measurements in EBR-I I " Nuc1. Technol. 25, 390 (1975).

60. G. G. Simons and A. P. 01 son, "Analyses and Measurements of Gamma-Ray Heating in the Demonstration Benchmark Plutonium-Fueled Critical Assembly," Nucl. Sci. Engng. 53, 176 (1974).

61. E. T. Boulette, D. R. Marr, and W. L. Bunch, "Analysis of the FTR/EMC Neutron Shielding Experiment," HEDL-TME 73-27 (1973). 
62. A. M. Weinberg and E. P. Wigner, The Physical Theory of Neutron Chain Reactors, Univ. of Chicago Press, Chicago, Illinois (1958).

63. D. Meneghetti and W. B. Loewenstein, "EBR-II Physics Experience" Proc. Int. Sym. on Physics of Fast Reactors, Vol. I, 175, Tokyo (1973).

64. D. Meneghetti and K. E. Phillips, "Energy Deposition in Iron and in Sodium by Elastic Moderation in EBR-II" ANL-RDP-44 (1975).

65. K. K. Mehta and A. M. Stadnyk, "Radiation Dosimetry in WR-1 Reactor, Part II. Ionization Chambers," AECL-3796 (1972). 


\section{DISTRIBUTION}

UC-79 Basic (186)

UC - $79 \mathrm{~b} \quad$ (42)

UC-79d (18)

ERDA/RL (2)

A. C. Fremling

R. E. Constant

ERDA/FFTF PO (5)

R. L. Ferguson

ERDA/HQ (2)

Director, RDD

HEDL (97)

G. J. Alkire

D. L. Arms trong

E. 0. Ballard

J. A. Basmajian

J. F. Bates

R. A. Bennett

J. D. Berger - EBR-II, Idaho

T. K. Bierlein

K. R. Birney

L. D. Blackburn

H. R. Brager

D. E. Brown

W. L. Bunch

C. A. Burgess

R. J. Cash

S. A. Chastain

J. A. Christensen

T. T. Claudson

B. L. Combs

C. M. Cox

G. E. Culley

R. E. Dahl, Jr.

J. W. Daughtry

D. G. Doran

E. A. Evans

E. R. Gilbert

F. A. Garner

J. E. Hanson

E. N. Heck

R. J. Hennig 


\section{DISTRIBUTION (Cont'd)}

HEDL

T. Hikido

J. J. Holmes

C. W. Hunter

J. E. Irvin

J. L. Jackson

R. J. Jackson

D. L. Johnson

W. C. Kinsel

L. S. Kellogg

R. L. Knecht

J. J. Laidler

G. A. Last

R. D. Leggett

E. P. Lippincott

W. W. Little, Jr.

(2)

A. J. Lovell

D. E. Mahagin

W. Y. Matsumoto

W. N. McElroy

F. S. Moore

R. P. Omberg

L. A. Pember

R. E. Peterson

A. L. Pitner

H. G. Powers

W. E. Roake

R. E. Schenter

J. 0. Schiffgens

D. P. Schively

F. A. Scott

W. F. Sheely

F. R. Shober

R. L. Simons

R. J. Slember

W. G. Spear

J. L. Straalsund

W. H. Sutherland

J. A. Ulseth

A. L. Ward

D. F. Washburn

J. W. Weber

H. H. Yoshikawa

Central Records \& Files (20)

Technical Communications 